\title{
On the appearance of internal wave attractors due to an initial or parametrically excited disturbance
}

\author{
Janis Bajars ${ }^{1}$, Jason Frank ${ }^{1} \dagger$ and Leo R. M. Maas ${ }^{2}$ \\ ${ }^{1}$ Centrum Wiskunde \& Informatica, PO Box 94079, 1090 GB Amsterdam, The Netherlands \\ ${ }^{2}$ Royal Netherlands Institute for Sea Research, PO Box 59, 1790 AB Texel, The Netherlands
}

(Received 26 March 2012; revised 8 July 2012; accepted 26 September 2012)

In this paper we solve two initial value problems for two-dimensional internal gravity waves. The waves are contained in a uniformly stratified, square-shaped domain whose sidewalls are tilted with respect to the direction of gravity. We consider several disturbances of the initial stream function field and solve both for its free evolution and for its evolution under parametric excitation. We do this by developing a structurepreserving numerical method for internal gravity waves in a two-dimensional stratified fluid domain. We recall the linearized, inviscid Euler-Boussinesq model, identify its Hamiltonian structure, and derive a staggered finite difference scheme that preserves this structure. For the discretized model, the initial condition can be projected onto normal modes whose dynamics is described by independent harmonic oscillators. This fact is used to explain the persistence of various classes of wave attractors in a freely evolving (i.e. unforced) flow. Under parametric forcing, the discrete dynamics can likewise be decoupled into Mathieu equations. The most unstable resonant modes dominate the solution, forming wave attractors.

Key words: geophysical and geological flows, internal waves

\section{Introduction}

Internal gravity waves in uniformly stratified fluids retain their frequency and consequently also their angle with respect to gravity upon reflection from an inclined boundary. Waves do change their wavelength, and become focused or defocused when reflecting from plane, inclined surfaces. Laboratory experiments confirm that when a container filled with a uniformly stratified fluid is excited vertically or horizontally, internal gravity waves appear, which become focused when reflecting from a sloping wall and converge towards a limit cycle, a so-called wave attractor (Maas \& Lam 1995; Maas et al. 1997; Hazewinkel et al. 2008). Energy propagates along the straight lines of the attractor, which are normal to the direction of phase propagation. Understanding the behaviour of internal waves in bounded domains may be important for explaining the mixing processes in ocean basins and lakes, and has relevance to astrophysics and fluid dynamics in general (Bühler \& Holmes-Cerfon 2011).

The ideal setting, considered above and used in typical laboratory and theoretical settings (including ours), assumes the fluid's stratification to be uniform, the domain's 
boundaries to be smooth and the setting to be two-dimensional. Non-uniform stratification, rough topography and three-dimensionality may, however, all lead to scattering of the internal wave field. Moreover, dissipation and nonlinear wave interaction limit the amplification of internal waves and might thus prohibit the ultimate localization of internal waves onto wave attractors.

Nevertheless, laboratory and numerical experiments have shown that wave attractors may be resilient to some of these perturbations. In the laboratory, attractors were shown to persist despite basins having non-uniform stratification, small-scale boundary corrugations (Hazewinkel et al. 2010) or being forced non-centrally in a three-dimensional (paraboloidal) domain (Hazewinkel, Grisouard \& Dalziel 2011). Numerically, attractors were obtained using multi-purpose numerical codes in idealized two-dimensional trapezoidal domains (Grisouard, Staquet \& Pairaud 2008), in threedimensional parabolic channel domains (Drijfhout \& Maas 2007) or in geometries mimicking realistically the Luzon Strait in the South China Sea (Tang \& Peacock 2010; Echeverri et al. 2011). Because of the interest in the dynamics of the Earth's liquid outer core and of stellar interiors, special attention has been devoted to wave attractors in spherical shells, where they are relevant to tidal dissipation and where they are resolved using spectral codes (e.g. Dintrans, Rieutord \& Valdettaro 1999; Tilgner 1999; Rieutord, Georgeot \& Valdettaro 2000).

But the actual relevance of internal wave attractors to real lakes, seas, oceans, atmospheres, the Earth's outer core, or planets and stars is unclear at present. Many factors may after all 'dilute' the ideal setting, and the evidence from direct observations is inconclusive or contradictory. Field observations in the small, $1 \mathrm{~km}$ wide stratified lake Mystic show that the horizontal velocity reaches its maximum at the sloping sides of the lake. This suggests that internal waves are steered towards a wave attractor instead of taking the shape of a seiche, a sloshing mode which would have its velocity maximum near the centre (Fricker \& Nepf 2000). Earlier lake observations revealed the dominance of high-wavenumber vertical modes, indicative of the presence of the small scales associated with an attractor (LaZerte 1980). The non-uniform stratification and presence of sheared background currents, all affecting internal wave ray paths, have been held responsible for the apparent absence of an attractor in the much larger Faroe-Shetland channel (Gerkema \& van Haren 2012). The absence of an attractor may, however, also be due to a mismatch between aspect ratio and the ratio of wave and stratification frequencies. Recent satellite observations of internal solitary waves suggest that wave attractors might actually have served as the amplification mechanism required to explain the enigmatic appearance of internal solitary waves from weak surface tides over a particular $80 \mathrm{~km}$ stretch of the Red Sea (da Silva et al. 2012). This seems to emphasize that higher spatial resolution of periodic internal wave fields is needed in in situ measurements.

Here we concentrate on an unsolved 'academic aspect', addressing the response of a uniformly stratified two-dimensional fluid to an initial perturbation in a basin whose shape breaks the reflection symmetry of internal gravity waves. The ansatz of a time-periodic, single-frequency (monochromatic) solution to the linearized internal gravity wave equations yields a wave equation in space with Dirichlet boundary conditions. This makes the problem quite unusual, as it is ill-posed due to nonuniqueness. The problem allows for weak solutions that can be solved using the method of characteristics or through a regularization technique (Swart et al. 2007). Via the method of characteristics one can study the limit behaviour of reflecting rays in bounded domains. The most generic asymptotic solution is an attractor, which is a finite closed orbit of rays within the domain. The particular structure of internal 
(a)

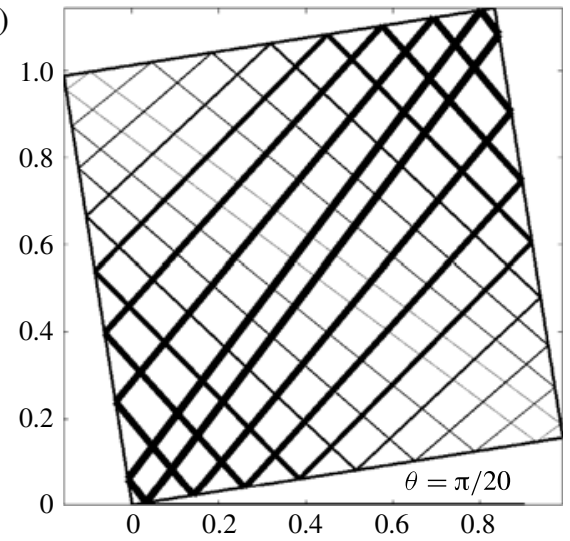

(b)

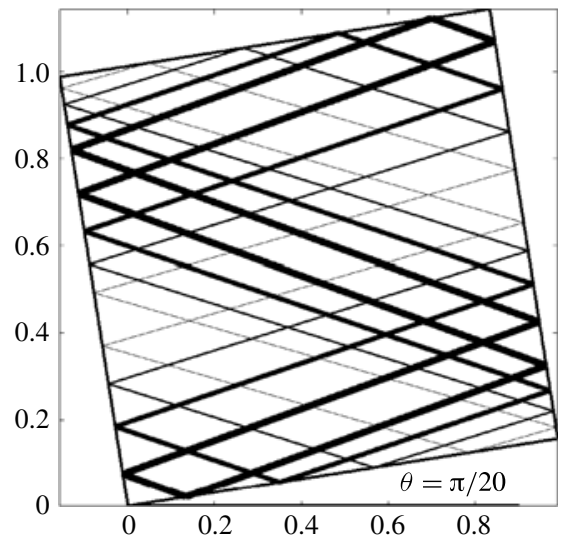

FIGURE 1. Limit cycle wave attractors corresponding to a discrete set of frequencies from the respective continuum ranges. Different line thicknesses correspond to distinct wave attractors: $(a)$ class of $(1,1)$ attractors; $(b)$ class of $(1,3)$ attractors.

gravity wave attractors in a tilted square domain depends on the rotation angle of the square $\theta$, the wave frequency $\omega$, and the stratification frequency $N_{f}$. A family of wave attractors is characterized by the number of reflections of a member-attractor from the boundary. By symmetry considerations, an attractor must reflect an equal number $n$ times with the top and bottom domain boundaries, and an equal number $m$ times with the left and right boundaries. Such an attractor is called an $(n, m)$ attractor. Figure 1 shows a discrete sample of the attractor geometries from the infinite classes of $(1,1)$ and $(1,3)$-attractors in a tilted square domain (see $\$ 2$ ).

Due to the ill-posedness of the monochromatic wave problem, we are motivated to study the initial value problem for internal gravity waves in a confined region. Alternatively, one could introduce viscosity, which regularizes the monochromatic wave problem, allowing for its approximate analytical solution (Ogilvie 2005). Lighthill (1996) considered the initial value problem for the evolution of a localized disturbance in an unbounded domain, deriving the dispersion relation and noting that vortical structures remain stationary after internal gravity waves have propagated away horizontally. In this paper we study internal waves in a stratified fluid filling a domain with solid walls, so that wave motion is trapped inside. We consider the simplest case that admits wave attractors: perturbations to a linearly stratified inviscid fluid, either freely evolving or parametrically excited. To guarantee that viscous effects play no role - not even implicitly via 'numerical diffusion' - we construct a numerical discretization that conserves total energy and symmetry in the absence of forcing, and study two idealized theoretical configurations: freely evolving (i.e. unforced) flow, and parametrically excited flow. We proceed with a normal-mode analysis of the discrete model. For the freely evolving case, we analyse the unforced initial boundary value problem, to show how linear dynamics is partitioned into normal modes for different classes of initial conditions. Figure 2 illustrates the free evolution from Fourier modes with wavenumbers $(1,1)$ and $(1,3)$, respectively. Evident in the plots at later times, we observe structures reminiscent of the full class of $(1,1)$ and $(1,3)$ attractors, suggesting a relationship between the Fourier modes and attractor geometries, for which we give some motivation. For the parametrically excited case, the normal-mode 
(a)

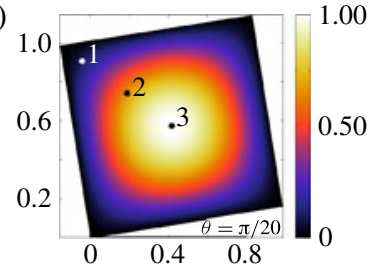

(d)

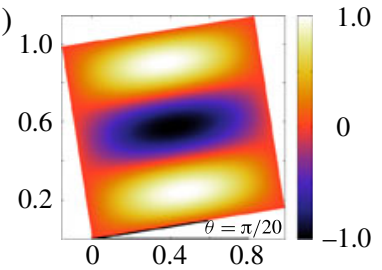

(b)

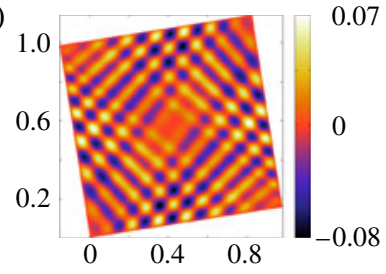

(e)

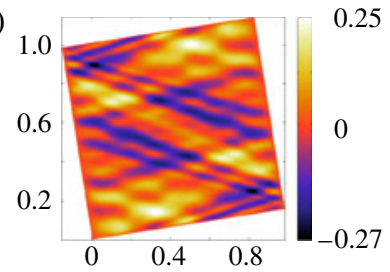

(c)

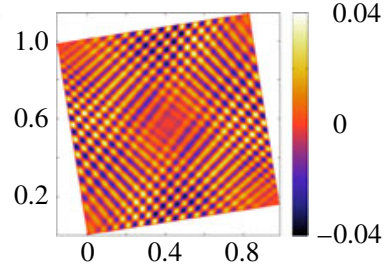

$(f)$

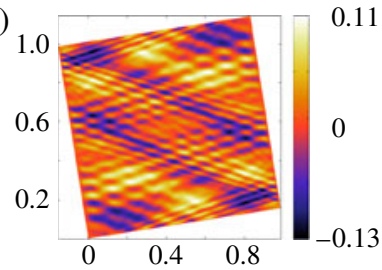

FIGURE 2. (Colour online) Evolution of the stream function in time from two distinct Fourier mode initial conditions.

analysis reveals that the flow may be decomposed into independent Mathieu equations, and that those modes whose associated frequencies lie within the resonance zones (Arnold tongues) will be amplified, forming a wave attractor.

It is important to note that the existence of a complete normal-mode decomposition for the discretized model contrasts sharply with the continuum model, for which the eigenspectrum is continuous and no such decomposition exists (Maas 2005). The continuous spectrum for the continuum model actually implies the existence of an uncountable infinity of time-periodic solutions, corresponding to the arbitrary definition of the boundary condition on the fundamental intervals, which we discuss. For the discretized system, the finite basis of normal modes is precisely the time-periodic solutions. The complete normal-mode decomposition for the discrete model is also non-robust with respect to viscous perturbation of the system. For the forced system with viscosity, the normal-mode basis becomes time-dependent, meaning the solution cannot be decomposed into scalar problems.

The paper is organized as follows. In $\S 2$ we recall the two-dimensional linear hydrostatic inviscid Euler-Boussinesq equations which govern internal gravity waves in stratified fluids, discuss monochromatic solutions in a tilted square domain, and review the Hamiltonian structure. In $\S 3$ we describe a structure-preserving finite difference discretization on the tilted square and present the normal-mode analysis of the discretized model in the unforced and forced cases. Using the symmetries of the discrete differential operators, we show that in both cases the dynamics may be projected onto an invariant basis of normal modes, such that they entirely decompose into independent scalar problems: harmonic oscillators in the unforced case or Mathieu equations in the forced case. In $\S 4$ we present numerical experiments of the unforced and forced models. We observe that an $(n, m)$ Fourier mode initial condition projects mostly onto the range of the associated $(n, m)$ attractor, explaining the similarities of figures 1 and 2. For the forced model we observe that if the initial condition has a non-trivial projection onto normal modes with amplified Mathieu dynamics, a wave attractor will emerge. Conclusions are summarized in $\S 5$. 


\section{Euler-Boussinesq equations}

\subsection{Internal gravity wave equations}

We consider a vertical slice domain $D \subset \mathbb{R}^{2}$ with boundary $\partial D$ and Cartesian coordinates $\boldsymbol{x}=(x, z)$, where $z$ is directed antiparallel to the direction of gravity, $\boldsymbol{g}$. We decompose the fluid density field and the pressure field as follows:

$$
\rho(x, z, t)=\rho_{0}+\bar{\rho}(z)+\rho^{\prime}(x, z, t), \quad p(x, z, t)=\bar{p}(z)+p^{\prime}(x, z, t),
$$

where $\rho_{0}$ is an average constant mean density and $\bar{\rho}(z)$ is a mean static density stratification, i.e. a monotonically decreasing function of $z$. The sum $\rho_{0}+\bar{\rho}(z)$ defines a stable background density field in hydrostatic balance with the pressure field $\bar{p}(z)$ :

$$
\partial_{z} \bar{p}=-g\left(\rho_{0}+\bar{\rho}(z)\right),
$$

where $g$ is the gravitational acceleration. The quantities $\rho^{\prime}(x, z, t)$ and $p^{\prime}(x, z, t)$ are small-amplitude perturbations about the (steady-state) background density and pressure fields.

In geophysical and astrophysical fluid dynamics it is common to treat the density field distinctly, defining both an 'inertial mass' and a 'gravitational mass'. The Boussinesq approximation consists of assuming a constant density value $\rho_{0}$ for the inertial mass in the momentum equation (from which the density may be consequently removed), while maintaining the full density $\rho$ for the gravitational mass. We enforce the inequality $\left|\rho^{\prime}\right| \ll|\bar{\rho}(z)| \ll \rho_{0}$ to justify the Boussinesq approximation. Such flows are termed 'buoyancy-driven'. The background stratification defines a stratification frequency, $N_{f}$ (the Brunt-Väisälä frequency), where $N_{f}^{2}=-g \rho_{0}^{-1} \mathrm{~d} \bar{\rho} / \mathrm{d} z$. In the following we assume that $N_{f}$ is a constant, i.e. the fluid is linearly stratified in the background density.

Wave focusing occurs when a boundary of the domain is inclined with respect to gravity. For this reason we assume that the coordinate system is rotated through an angle $0 \leqslant \theta \leqslant \pi / 4$. With the above considerations in mind, the inviscid linear Euler-Boussinesq equations describing the propagation of perturbations in this rotated frame read:

$$
\begin{aligned}
\partial_{t} \boldsymbol{u} & =-\nabla \hat{p}+b \hat{\boldsymbol{k}}(\theta), \\
\partial_{t} b & =-N_{f}^{2} \boldsymbol{u} \cdot \hat{\boldsymbol{k}}(\theta), \\
\nabla \cdot \boldsymbol{u} & =0 \\
\boldsymbol{u} \cdot \hat{\boldsymbol{n}} & =0 \quad \text { on } \partial D,
\end{aligned}
$$

where $\boldsymbol{u}=(u, w)$ is a velocity field in the $x$ and $z$ direction respectively (now tilted relative to the original direction), $\hat{p}=\rho_{0}^{-1} p^{\prime}$ is scaled pressure with respect to the mean constant density, $b=-g \rho^{\prime} \rho_{0}^{-1}$ is the buoyancy, $\hat{\boldsymbol{k}}(\theta)=(\sin \theta, \cos \theta)$ is the unit vector in the direction opposite to gravity and $\hat{\boldsymbol{n}}$ is the unit outward normal to the boundary $\partial D$.

In two dimensions it is convenient to consider the stream function formulation of the Euler-Boussinesq equations (2.3)-(2.6). The divergence-free condition (2.5) allows us to define a stream function $\psi$ on $D$ such that

$$
u=-\partial_{z} \psi, \quad w=\partial_{x} \psi .
$$

By taking the curl of the momentum equation (2.3) we eliminate the pressure from (2.3), obtaining the two-dimensional linear inviscid Euler-Boussinesq equations in 
stream function formulation:

$$
\begin{aligned}
\partial_{t} q & =-\partial_{x} b \cos \theta+\partial_{z} b \sin \theta, \\
\partial_{t} b & =-N_{f}^{2}\left(\partial_{x} \psi \cos \theta-\partial_{z} \psi \sin \theta\right), \\
q & =-\Delta \psi \\
\psi & =0 \quad \text { on } \partial D,
\end{aligned}
$$

where $q=\partial_{z} u-\partial_{x} w$ is vorticity.

The model (2.8)-(2.11) is a system of partial differential equations that conserves total energy:

$$
\mathscr{H}=\frac{1}{2} \int_{D}\left(\nabla \psi \cdot \nabla \psi+\frac{1}{N_{f}^{2}} b^{2}\right) \mathrm{d} \boldsymbol{x},
$$

equal to the sum of kinetic and potential energies.

\subsection{Forcing}

Wave attractors are generated by periodically forcing a stratified fluid in a domain with inclined boundaries. In the ocean, the forcing is primarily tidal forcing. In laboratory experiments (Maas et al. 1997; Lam \& Maas 2008), wave attractors were generated by vertically oscillating a container with a sloping wall. To incorporate such parametric excitation (McEwan \& Robinson 1975) (2.8) is modified by multiplication with a time-dependent function $\alpha(t)$ to obtain

$$
\partial_{t} q=\alpha(t)\left(-\partial_{x} b \cos \theta+\partial_{z} b \sin \theta\right)
$$

An alternative approach is external excitation, e.g. a horizontal oscillation of the container, for which time-dependent terms may be added to (2.8) and (2.9) (Ogilvie 2005), or by means of boundary forcing (Grisouard et al. 2008).

Vertical oscillation of the container can be viewed as time-dependent modulation of the gravitational parameter $g$, which originally enters the momentum equation, and should thus be present only in the vorticity equation (2.8). Hence, we can realize this kind of forcing as parametric excitation with

$$
\alpha(t)=1-\epsilon \cos (2 \omega t),
$$

where $\epsilon$ is a positive constant smaller than one and $2 \omega$ is the forcing frequency.

\subsection{Dispersion properties of internal gravity waves}

Consider a time-periodic solution

$$
\psi(x, z, t)=\Psi(x, z) \mathrm{e}^{-\mathrm{i} \omega t}, \quad b(x, z, t)=B(x, z) \mathrm{e}^{-\mathrm{i} \omega t} .
$$

Substituting the above ansatz into (2.8)-(2.10), eliminating $B$ and taking $\theta=0$ without loss of generality yields

$$
\partial_{z z} \Psi-\frac{\left(N_{f}^{2}-\omega^{2}\right)}{\omega^{2}} \partial_{x x} \Psi=0,
$$

which is recognized as a wave equation when $\omega^{2}<N_{f}^{2}$ for the scalar state variable $\Psi$. In other words, internal gravity waves are spatially governed by the wave equation. Substituting the plane wave

$$
\Psi(x, z)=a \exp \left(\mathrm{i}\left(\kappa_{x} x+\kappa_{z} z\right)\right)
$$


into (2.16), where $a$ is the amplitude and $\kappa_{x}$ and $\kappa_{z}$ are wavenumbers, yields the dispersion relation

$$
\omega^{2}=N_{f}^{2} \frac{\kappa_{x}^{2}}{\kappa_{x}^{2}+\kappa_{z}^{2}}=N_{f}^{2} \cos ^{2} \phi
$$

the last equality of which follows from the polar coordinate description of the wavenumber vector $\kappa=|\kappa|(\cos \phi, \sin \phi)$, where $|\boldsymbol{\kappa}|$ is the wavenumber magnitude and $\phi$ its direction. Hence, $\omega^{2} \leqslant N_{f}^{2}$ and the frequencies of internal gravity waves are bounded by the stratification frequency $N_{f}$. It is also apparent that the wave frequency is independent of the wavenumber magnitude and depends only on its angle $\phi$. Consequently an incident wave retains its propagation direction upon reflection from a plane surface independent of the slope of the surface, leading to monoclinic (single-angled) waves. A wave does, in general, change its wavelength, and can become focused or defocused upon reflection from an inclined boundary. It is well known that the wave phase travels in the phase velocity direction $c_{p}=\omega \boldsymbol{\kappa} /|\boldsymbol{\kappa}|^{2}$ and wave packet energy is transported by the group velocity $c_{g}=\nabla_{\kappa} \omega$, (Whitham 1999). The internal wave group velocity vector $c_{g}$ and phase velocity vector $c_{p}$ are mutually perpendicular, i.e. $\boldsymbol{c}_{\boldsymbol{g}} \cdot \boldsymbol{c}_{\boldsymbol{p}}=0$. Hence internal waves propagate energy parallel to the wave crests and troughs (i.e. along them).

\subsection{Monochromatic wave solutions in a tilted square}

The wave equation (2.16) with Dirichlet boundary conditions (2.11) is formally an ill-posed problem (Swart et al. 2007). One not only finds a trivial solution $\psi \equiv 0$, but there exist infinitely many solutions. For example, the hyperbolic wave equation (2.16) can be solved on a non-inclined $(\theta=0)$ rectangular domain $(x, z) \in[0,1] \times[0, \ell]$ by separation of variables. The function

$$
\Psi=A_{n, m} \sin (n \pi x) \sin (m \pi z / \ell)
$$

satisfies the hyperbolic equation (2.16) and boundary condition (2.11) provided that

$$
\ell=\sqrt{\frac{\omega^{2}}{N_{f}^{2}-\omega^{2}}} \frac{m}{n}
$$

Replacing integer $(n, m)$ in $(2.20)$ by $(j n, j m)$ leaves $\ell$ unchanged, and for integer $j, \Psi$ still vanishes at the boundaries. In this non-inclined case there is a countably infinite set of solutions to the wave equation (2.16); in the inclined case this set is not countable, resulting in the ill-posedness.

The general solution of the wave equation (2.16) is given by

$$
\Psi(x, z)=f(x-\gamma z)-g(x+\gamma z), \quad \gamma=\sqrt{\frac{N_{f}^{2}-\omega^{2}}{\omega^{2}}},
$$

for arbitrary functions $f$ and $g$. Hence the function $g$ is constant along a characteristic line $x+\gamma z=$ const., and likewise $f$ is constant along lines $x-\gamma z=$ const. Furthermore, the Dirichlet boundary condition, $\Psi=0$, implies that $f \equiv g$ on the boundary. Therefore, from any point $p$ in the domain, one can define an orbit, consisting of a characteristic passing through $p$ and the infinite sequence of successive reflections of that characteristic in both forward and backward orientation upon which $f$ and $g$ are alternately constant. Such a sequence of characteristics will be referred to as a characteristic orbit. Two characteristic orbits intersect at each point $p$ in the interior 
of $D$, and the difference $f-g$ determines the stream function at $p$. One can follow characteristic orbits that intersect at $p$ until they reach a boundary segment upon which the function $f=g$. The problem of determining a well-posed monochromatic solution is reduced to that of identifying a minimal set of distinct intervals, the so-called fundamental intervals, on the boundary where the functions $f$ and $g$ may be prescribed (see Maas \& Lam 1995).

For this paper we will study internal waves in a tilted square domain. In the tilted unit square the topology of a complete characteristic orbit passing through a point depends on the angle of tilt $\theta$ and the ratio of wave frequency to stratification frequency $\omega / N_{f}$. In the subcritical case all characteristic orbits asymptotically approach diagonally opposite corners of the square. This occurs when the characteristic slopes $\pm \gamma$ are both either larger or smaller than the inclination of both horizontal and vertical boundaries. In the supercritical case one can distinguish an additional three types of limit behaviour: periodic, ergodic and limit cycle orbits (John 1941; Kopecz 2006). In the periodic case all characteristic orbits reflect from the boundary at a finite number of points, the fundamental intervals collapse onto one another, and the characteristic orbit through every point is periodic. In the ergodic case, the characteristic orbit through any point passes arbitrarily close to every other point in the domain, the fundamental interval shrinks to a single point, and the stream function then necessarily vanishes, implying no flow. However, the most generic case of limit behaviour of the characteristic orbits is an attractor or limit cycle, i.e. one or more distinct periodic orbits that attracts a neighbourhood of itself. Such attractors are characterized by the number of boundary reflections from the horizontal and vertical boundaries. Considering the symmetry of the top and bottom boundary and of the two side boundaries, we denote by $(n, m)$ an attractor having $n$ reflections from the boundary on the upper side of the square and $m$ reflections from the left side of the square. The overall number of reflections with the boundary $(2 n+2 m)$ is called the attractor's period. In the unit square domain all attractors are globally attracting.

The choice of the fundamental intervals on the boundary and the functions prescribed on them is not unique. In the subcritical case it is sufficient to prescribe only one interval between two successive characteristic reflections from the boundary. In the ergodic case the solution may be prescribed at only one point on the boundary yielding the trivial solution $\psi \equiv 0$ of the wave equation (2.16) due to the zero Dirichlet boundary conditions (2.11). For the periodic and attractor cases one must prescribe one or two intervals on one of the square's boundaries, respectively. For a complete discussion see Maas \& Lam (1995).

Let us take a closer look at periodic solutions and limit cycles. The experimental variables are the wave frequency $\omega$, stratification frequency $N_{f}$ and rotation angle of the square $\theta$. In the periodic solution regime, all orbits correspond to odd-even pairs $(2 n, 2 m+1)$ or $(2 n+1,2 m)$. But the periodic regime is non-robust with respect to perturbations in domain geometry. In the tilted square domain these solutions occur only for a discrete set of frequencies. In contrast the limit cycle attractors persist over a continuous range of frequencies, hence are robust with respect to frequency perturbations. In the simplest periodic case the characteristic orbit emanating from, say, the lower left corner of the square will precisely intersect the lower right corner after making $n$ successive reflections from the top of the square, or will intersect the upper left corner after $m$ successive reflections from the right side of the square. In both such situations we have analytic expressions relating the wave frequency $\omega$, stratification 

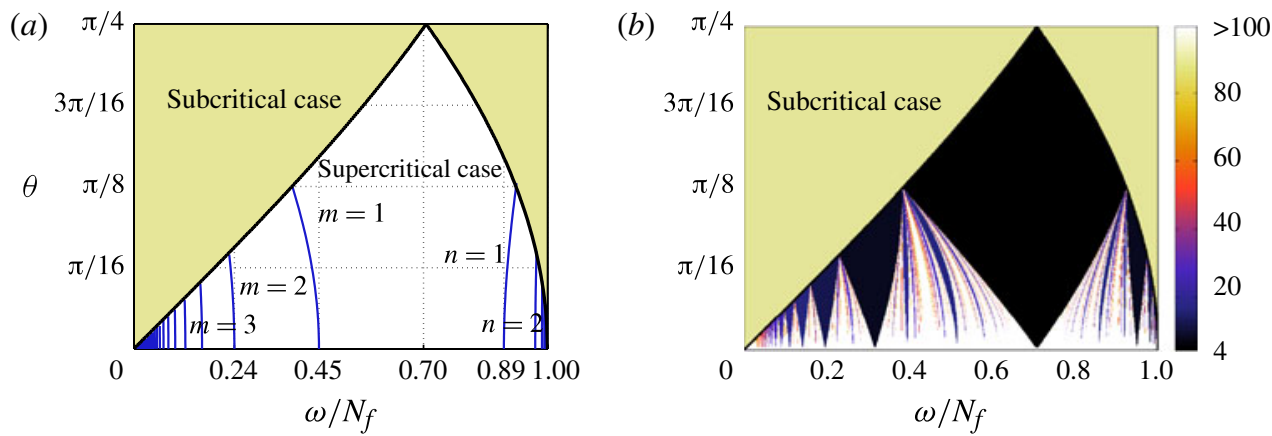

FIgure 3. (Colour online) Parameter space for monochromatic solutions: (a) loci in parameter space corresponding to periodic solutions $(2 n, 1)$ and $(1,2 m) ;(b)$ limit cycle attractor period, indicated by shading.

frequency $N_{f}$ and rotation angle of the square $\theta$ :

$$
\begin{aligned}
& \cot \left(\theta+\tan ^{-1} \sqrt{\frac{\omega^{2}}{N_{f}^{2}-\omega^{2}}}\right)-\cot \left(\theta-\tan ^{-1} \sqrt{\frac{\omega^{2}}{N_{f}^{2}-\omega^{2}}}\right)=\frac{1}{n}, \\
& \tan \left(\theta+\tan ^{-1} \sqrt{\frac{\omega^{2}}{N_{f}^{2}-\omega^{2}}}\right)-\tan \left(\theta-\tan ^{-1} \sqrt{\frac{\omega^{2}}{N_{f}^{2}-\omega^{2}}}\right)=\frac{1}{m}
\end{aligned}
$$

respectively. Hence these periodic solutions are indicated as $(2 n, 1)$ and $(1,2 m)$ with periods $2(2 n+1)$ and $2(2 m+1)$, respectively. Similar periodic solutions can be computed when the characteristic orbits have multiple reflections from both the left and top boundaries, and geometries $(2 n, 2 m+1)$ or $(2 n+1,2 m)$.

Figure 3 illustrates the parameter space $\omega / N_{f}$ versus $\theta$. The bold line separates subcritical and supercritical regimes. Within the supercritical region of figure $3(a)$, we indicate the loci of parameter values corresponding to periodic solutions of the classes $(2 n, 1)$ and $(1,2 m)$. Note that for a given rotation angle $\theta$, the periodic solutions correspond to discrete values of $\omega / N_{f}$. Limit cycle solutions are indicated in figure $3(b)$, where the shading denotes the period of the attractor. Periodic solutions (figure $3 a$ ) are found where the attractor period (figure $3 b$ ) approaches infinity.

Figure 4 shows solutions of the monochromatic wave $(2.16)$ for the $(1,1)$ and $(1,3)$ attractor cases and for the $(1,2)$ periodic case, for specific values of $\theta$ and $\omega / N_{f}$. In figure $4(a, b)$ we show two typical members from the respective continuum ranges of limit cycle solutions. In both cases one can observe a self-similar structure approaching the attractor. The solutions were constructed using the method of characteristics; on the fundamental intervals we prescribe two cosines with an offset at the chosen intervals. For a square-shaped attractor in a trapezoidal geometry, a free wave solution possessing a logarithmic self-similar Fourier spectrum was computed analytically (Maas 2009).

\section{Numerical discretization and linear analysis}

In this section we describe our discrete model equations and show that in the special case of linear inviscid flow, the dynamics decouples into scalar oscillators. 

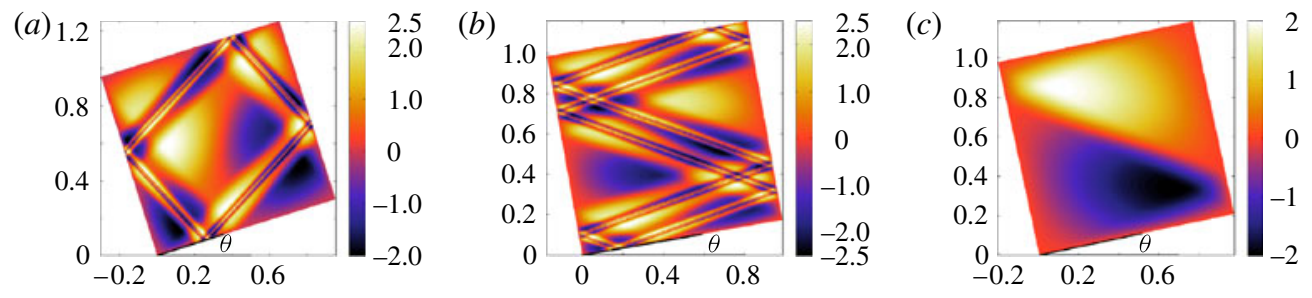

FIGURE 4. (Colour online) Monochromatic stream function solutions: $(a)$ within the $(1,1)$ attractor frequency range $\left(\omega / N_{f}=0.74, \theta=7 \pi / 72\right) ;(b)$ within the $(1,3)$ attractor frequency range $\left(\omega / N_{f}=0.34, \theta=\pi / 18\right) ;(c)$ the unique $(1,2)$ periodic solution $\left(\omega / N_{f}=0.43\right.$, $\theta=\pi / 15)$.

\subsection{Fourier analysis of the continuum model, non-tilted}

For a non-tilted square domain $(\theta=0)$, the initial boundary value problem for the linear Euler-Boussinesq equations (2.8)-(2.11) with initial conditions $\psi_{0}(x, z)$ and $b_{0}(x, z)$ and zero Dirichlet boundary conditions (2.11) can be solved analytically using separation of variables. The solution is

$$
\psi(x, z, t)=\sum_{n, m=1}^{\infty} \psi_{n, m}(x, z) \frac{\mathrm{d}}{\mathrm{d} t} T_{n, m}(t), \quad b(x, z, t)=-N_{f}^{2} \sum_{n, m=1}^{\infty} \partial_{x} \psi_{n, m}(x, z) T_{n, m}(t),
$$

where $\psi_{n, m}(x, z)=\sin (n \pi x) \sin (m \pi z)$ are Fourier modes on the unit square, i.e. the eigenfunctions of the operators $\partial_{x x}$ and $\partial_{z z}$ under the given boundary conditions, and $T_{n, m}$ is a solution to the simple harmonic oscillator equation

$$
\frac{\mathrm{d}^{2}}{\mathrm{~d} t^{2}} T_{n, m}=-\omega_{n, m}^{2} T_{n, m}, \quad \omega_{n, m}^{2}=N_{f}^{2} \frac{n^{2}}{n^{2}+m^{2}},
$$

with the frequencies given by the dispersion relation (2.18).

The total energy functional (2.12) of the general solution in the form (3.1) is

$$
\mathscr{H}=\frac{\pi}{8} \sum_{n, m=1}^{\infty}\left[\left(n^{2}+m^{2}\right)\left(\frac{\mathrm{d}}{\mathrm{d} t} T_{n, m}\right)^{2}+N_{f}^{2} n^{2} T_{n, m}^{2}\right]=\sum_{n, m=1}^{\infty} \mathscr{H}_{n, m},
$$

where for each $(n, m)$, the term in square brackets, $\mathscr{H}_{n m}$, is the independently conserved Hamiltonian of (3.2). Note that there is no coupling between wavenumbers. The initial conditions may be projected onto the Fourier modes, but each mode evolves independently, and there is no energy exchange between modes.

The situation for $\theta \neq 0$ is very different. The initial boundary value problem (2.8)-(2.11) cannot be solved analytically by the method of separation of variables as was done above. The eigenfunctions in the tilted case correspond to the ill-posed solutions of (2.16), and have no simple representation. However, as we show in the next section, the numerical discretization does admit a normal-mode analysis.

\subsection{Energy-conserving numerical discretization and analysis}

Making use of the Hamiltonian structure of (2.8)-(2.11), we construct in appendix A an energy-preserving numerical discretization. Discretizing in space while leaving time 
continuous yields the following system of linear ordinary differential equations (cf. (A 27)-(A 29)):

$$
\begin{aligned}
-\boldsymbol{L} \frac{\mathrm{d} \boldsymbol{\psi}}{\mathrm{d} t} & =\alpha(t)\left(\boldsymbol{D}_{x}^{\mathrm{T}} \boldsymbol{M}_{z} \boldsymbol{b} \cos \theta-\boldsymbol{D}_{z}^{\mathrm{T}} \boldsymbol{M}_{x} \boldsymbol{b} \sin \theta\right), \\
\frac{\mathrm{d} \boldsymbol{b}}{\mathrm{d} t} & =-N_{f}^{2}\left(\boldsymbol{M}_{z}^{\mathrm{T}} \boldsymbol{D}_{x} \boldsymbol{\psi} \cos \theta-\boldsymbol{M}_{x}^{\mathrm{T}} \boldsymbol{D}_{z} \boldsymbol{\psi} \sin \theta\right),
\end{aligned}
$$

where $\boldsymbol{\psi} \in \mathbb{R}^{M}$ and $\boldsymbol{b} \in \mathbb{R}^{N}, M<N$, are vectors containing the values of $\psi$ and $b$ at (staggered) grid positions. The finite difference matrices $\boldsymbol{M}_{x}, \boldsymbol{M}_{z}, \boldsymbol{D}_{x}, \boldsymbol{D}_{z}$ and $\boldsymbol{L}$, defined in $\S$ A.1, represent discretized mean $\left(M_{*}\right)$, difference $\left(D_{*}\right)$ and Laplacian $(L)$ operators, and superscript $\mathrm{T}$ denotes the transpose. Here we have introduced the factor $\alpha(t)$, which allows us to add forcing by means of parametric excitation. Introducing the matrix $\boldsymbol{K}=\boldsymbol{D}_{x}^{\mathrm{T}} \boldsymbol{M}_{z} \cos \theta-\boldsymbol{D}_{z}^{\mathrm{T}} \boldsymbol{M}_{x} \sin \theta$, this system can be written in matrix form as

$$
\left[\begin{array}{cc}
-\boldsymbol{L} & 0 \\
0 & \boldsymbol{I}
\end{array}\right] \frac{\mathrm{d}}{\mathrm{d} t}\left(\begin{array}{l}
\boldsymbol{\psi} \\
\boldsymbol{b}
\end{array}\right)=\left[\begin{array}{cc}
0 & \alpha(t) \boldsymbol{K} \\
-N_{f}^{2} \boldsymbol{K}^{\mathrm{T}} & 0
\end{array}\right]\left(\begin{array}{l}
\boldsymbol{\psi} \\
\boldsymbol{b}
\end{array}\right)
$$

By construction, when forcing is absent $(\alpha \equiv 1)$ the discretization possesses a first integral, the discrete Hamiltonian $H$ (A 18), which approximates the total energy (2.12), i.e.

$$
H=\frac{1}{2}\left(-\boldsymbol{\psi}^{\mathrm{T}} \boldsymbol{L} \psi+\frac{1}{N_{f}^{2}} \boldsymbol{b}^{\mathrm{T}} \boldsymbol{b}\right) \Delta x \Delta z
$$

In appendix B we derive the normal-mode bases $\boldsymbol{X}=\left(\boldsymbol{X}_{1}, \ldots, \boldsymbol{X}_{M}\right)$ and $\boldsymbol{Y}=$ $\left(\boldsymbol{Y}_{1}, \ldots, \boldsymbol{Y}_{N}\right)$, in which $\boldsymbol{\psi}$ and $\boldsymbol{b}$ are expressed as (cf. (B 12))

$$
\psi=X \tilde{\psi}, \quad b=Y \tilde{b}
$$

In the new basis, the system (3.6) decouples into $M$ second-order problems:

$$
\begin{aligned}
\frac{\mathrm{d}^{2}}{\mathrm{~d} t^{2}} \tilde{\psi}_{i} & =-\alpha(t) \omega_{i}^{2} \tilde{\psi}_{i}+\dot{\alpha}(t) \omega_{i} \tilde{b}_{i}, \\
\frac{\mathrm{d}^{2}}{\mathrm{~d} t^{2}} \tilde{b}_{i} & =-\alpha(t) \omega_{i}^{2} \tilde{b}_{i},
\end{aligned}
$$

for $i=1, \ldots, M$, plus the trivial dynamics $\left(\mathrm{d}^{2} / \mathrm{d} t^{2}\right) \tilde{b}_{i}=0, i=M+1, \ldots, N$.

When forcing is absent, $\alpha(t) \equiv 1$, the dynamics further decouples into $2 M$ independent harmonic oscillators

$$
\frac{\mathrm{d}^{2}}{\mathrm{~d} t^{2}} \tilde{\psi}_{i}=-\omega_{i}^{2} \tilde{\psi}_{i}, \quad \frac{\mathrm{d}^{2}}{\mathrm{~d} t^{2}} \tilde{b}_{i}=-\omega_{i}^{2} \tilde{b}_{i}, \quad i=1, \ldots, M
$$


In particular, the total energy can be expressed as the sum of the harmonic oscillator energies

$$
\left.\begin{array}{l}
H=\sum_{i=1}^{M} H_{i}^{\psi}+H_{i}^{b}, \\
H_{i}^{\psi}=\frac{1}{2}\left[\left(\frac{\mathrm{d} \tilde{\psi}_{i}}{\mathrm{~d} t}\right)^{2}+\omega_{i}^{2} \tilde{\psi}_{i}^{2}\right], \\
H_{i}^{b}=\frac{1}{2}\left[\left(\frac{\mathrm{d} \tilde{b}_{i}}{\mathrm{~d} t}\right)^{2}+\omega_{i}^{2} \tilde{b}_{i}^{2}\right],
\end{array}\right\}
$$

each of which is a conserved quantity.

Remark. In $\S 2$ we saw that there are infinitely many monochromatic wave solutions to the linearized Euler-Boussinesq equations, corresponding to an arbitrary specification of the solution on a fundamental interval. For the discretized equations, of course, there can be only a finite number of periodic solutions, each corresponding to a normal mode of the discretization matrix. This situation is analogous to the case of the advection equation $\rho_{t}+u \rho_{x}=0$ on a periodic domain, for which any initial condition $\rho(x, 0)=f(x)$ is periodic in time. Upon numerical discretization of this equation, the dispersion relation is altered, an arbitrary initial condition may be expanded in normal modes, and each of these evolves with a different phase speed, causing artificial dispersion. Only the (finite countable) normal modes themselves are periodic.

When parametric forcing is present in (3.10), i.e. $\alpha(t)=1-\epsilon \cos 2 \omega t$, the buoyancy modes evolve independently according to the Mathieu equation

$$
\frac{\mathrm{d}^{2}}{\mathrm{~d} t^{2}} \tilde{b}_{i}=-(1-\epsilon \cos (2 \omega t)) \omega_{i}^{2} \tilde{b}_{i}
$$

The Mathieu equation supports resonance zones in parameter space for which the solution grows unbounded in magnitude, as well as stable (non-resonant) zones for which the solution remains bounded for all time. The first and most important instability region originates at the subharmonic frequency $\omega$ of the driving frequency $2 \omega$ (see Arnold 1989).

\subsection{Dynamics of the Mathieu equation}

Rescaling time with respect to the stratification frequency $N_{f}$, i.e. $t^{\prime}=N_{f} t$, in (3.13) yields, dropping primes,

$$
\frac{\mathrm{d}^{2}}{\mathrm{~d} t^{2}} \tilde{b}_{i}=-\left(1-\epsilon \cos \left(2 \frac{\omega}{N_{f}} t\right)\right) \frac{\omega_{i}^{2}}{N_{f}^{2}} \tilde{b}_{i}
$$

where $\omega_{i}^{2} / N_{f}^{2} \leqslant 1$ from the dispersion relation. For a given value of the (normalized) first subharmonic forcing frequency $\left|\omega / N_{f}\right| \leqslant 1$ we are interested in knowing for which normal-mode frequencies $\omega_{i} / N_{f}$ and forcing amplitude $\epsilon$ (3.14) and (3.13) support resonances.

Introducing a second time transformation, $t^{\prime}=\omega N_{f}^{-1} t$, we write the scalar Mathieu (3.14) in the general form

$$
\frac{\mathrm{d}^{2}}{\mathrm{~d} t^{2}} \beta+(a-2 q \cos (2 t)) \beta=0,
$$



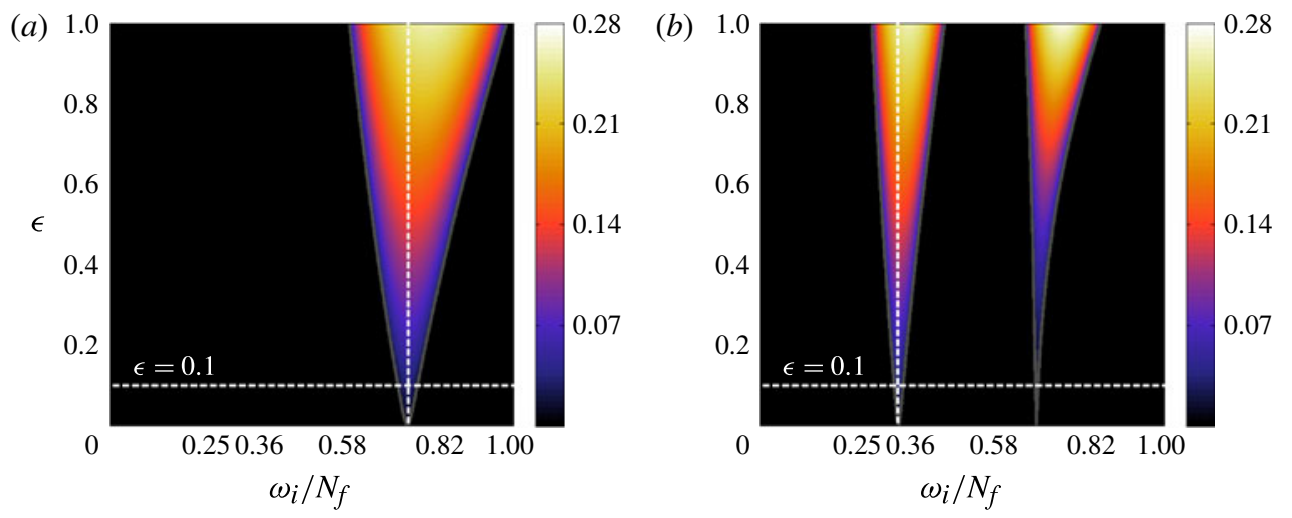

FIGURE 5. (Colour online) Instability tongues of the Mathieu equation, the shading denoting the magnitude of Floquet exponent $\operatorname{Re} \mu$, as a function of normal-mode frequency $\omega_{i} / N_{f}$ for different forcing amplitudes $\epsilon$. (a) Subharmonic forcing frequency $\omega / N_{f}=0.74$, one instability tongue in the computation of the $(1,1)$ attractor. $(b)$ Subharmonic forcing frequency $\omega / N_{f}=0.34$, two instability tongues in the computation of the $(1,3)$ attractor. The vertical and horizontal dashed lines indicate forcing frequencies and amplitudes respectively.

where $\beta=\tilde{b}_{i}, a=\omega_{i}^{2} / \omega^{2} \leqslant N_{f}^{2} / \omega^{2}$ and $q=(\epsilon / 2) \omega_{i}^{2} / \omega^{2} \leqslant(\epsilon / 2) N_{f}^{2} / \omega^{2}$ for a given normal mode $i$. According to the Floquet multiplier theorem, the Mathieu equation for fixed $a$ and $q$ admits a complex-valued general solution of the form

$$
\beta(t)=c_{1} \mathrm{e}^{\mu t} P(a, q, t)+c_{2} \mathrm{e}^{-\mu t} P(a, q,-t),
$$

where $\mu \neq 0$ is a complex Floquet exponent and $P(a, q, t)$ is a complex-valued, $\pi$ periodic, special function, i.e. $P(a, q, t+\pi)=P(a, q, t)$. If $\operatorname{Re} \mu=0$, the solution $\beta(t)$ is bounded for all time. If $\operatorname{Re} \mu \neq 0$, the amplitude of the oscillations grows exponentially. For the degenerate case $\mu=0$, the solutions are linearly dependent and the amplitude grows linearly in time.

To determine the Floquet exponent $\mu$ we note that by taking as initial conditions $\beta(0)=1$ and $\dot{\beta}(0)=0$ one finds $c_{1}=c_{2}=(2 P(a, q, 0))^{-1}$, and hence the solution at time $t=\pi$ is

$$
\beta(\pi)=\cosh \mu \pi \text {. }
$$

Therefore $\mu$ can be estimated by solving (3.15) numerically on the interval $[0, \pi]$. For a given forcing $\omega / N_{f}$, we solve for $\mu$ numerically using the Störmer-Verlet method (Hairer, Lubich \& Wanner 2006) over a discrete set of values $\epsilon \in[0,1]$ and $\omega_{i} / N_{f} \in[0,1]$.

Our goal is to investigate the emergence of the two internal wave attractors presented in $\S 2$ by use of the parametric excitation mechanism described above. We expect that after an initial transient phase, the solution will be dominated by those normal modes having positive Floquet exponents. We fix $\epsilon=0.1$ and choose forcing frequencies $2 \omega / N_{f}$, whose subharmonics excite the patterns in figure 4, i.e. we choose $\omega / N_{f}=0.74$ or $\omega / N_{f}=0.34$, respectively. In figure 5 we plot the real part of the Floquet exponent $\mu$ as a function of normal-mode frequency $\omega_{i} / N_{f} \in[0,1]$ (regarding $\omega_{i} / N_{f}$ as a continuous variable). For these two cases we obtain the instability tongues shown in figures $5(a)$ and $5(b)$, respectively. Figure 5(a) shows the real part of the Floquet exponent $\mu$ for subharmonic forcing frequency $\omega / N_{f}=0.74$. The resonant 
instability tongue originates at $\omega_{i} / N_{f}=0.74$, and superharmonic resonances $\left(n \omega / N_{f}\right.$, $n=2,3, \ldots$ ) are absent because they fall outside the admissible range of normal-mode frequencies. Figure 5(b) shows $\operatorname{Re} \mu$ for subharmonic forcing frequency $\omega / N_{f}=0.34$. The first resonant instability tongue then originates at $\omega_{i} / N_{f}=0.34$, and also the first superharmonic resonance at $\omega_{i} / N_{f}=2 \omega / N_{f}=0.68$ falls within the admissible range of normal-mode frequencies. For a given value of subharmonic forcing frequency $\omega / N_{f}$, the rotation angle $\theta \in[0, \pi / 4]$ determines the type of limit behaviour observed, e.g. an attractor or a periodic solution: see figure 3 .

Since the forced internal wave equations (3.4)-(3.5) can be decomposed into the Mathieu-type equations (3.9)-(3.10), the theory of Mathieu equations suggests that, depending on the values of the Floquet exponent, there will be resonant normal modes which will grow exponentially in time and there will be other modes which will stay bounded. The presence of resonant normal modes is dependent on the initial conditions. If a particular initial condition is such that its projection onto normal modes has no components within resonant zones of the Mathieu equation, then the solution of the forced linear internal wave equations (3.4)-(3.5) will stay bounded for all times. Hence the choice of initial conditions for computations is not arbitrary. The analysis in $\S 4.1$ of the system's response to different initial conditions in the unforced, undamped linear case suggests that the natural choice for finding $(1,1)$ and $(1,3)$ attractors would be initial conditions $\psi_{1,1}$ and $\psi_{1,3}$, respectively. This implies that there will be resonant normal modes.

\section{Numerical experiments}

\subsection{Freely evolving flow}

Armed with the theory of internal gravity wave attractors in a tilted square from $\S 2$ and the structure-preserving discretization of the Euler-Boussinesq equations in the stream function formulation from $\S 3$, we study the initial boundary value problem. Since we consider the inviscid equations, the system does not depend on spatial scales and time can be rescaled with respect to stratification frequency $N_{f}$ to cast the system in dimensionless form. As we will see in the following, the response of the system will depend on tilt angle $\theta$ and on the choice of the initial conditions.

We study the response of the system with the Fourier mode initial conditions:

$$
\psi_{0}(x, z)=\psi_{n, m}(x, z), \quad b_{0}(x, z) \equiv 0, \quad(n, m)=(1,1),(1,2),(1,3) .
$$

These initial conditions correspond to low-wavenumber smooth functions. When $\theta=0$ the Fourier modes are eigenfunctions, as described in $\$ 3.1$, and all three initial conditions result in single-frequency standing wave solutions whose frequency is determined by the dispersion relation (2.18). When $\theta \neq 0$, i.e. the domain is tilted by the angle $\theta$ or the direction of gravity is changed, the Fourier modes are no longer eigenfunctions, and we observe a different response from the system for initial conditions (4.1).

In all three numerical examples we use the same numerical parameters and parameter values. We compute to final time $T_{\text {end }}=400$ with time step $\tau=0.05$. The spatial mesh sizes in both space dimensions are equal, $\Delta x=\Delta z=2 \times 10^{-3}$. We fix the stratification frequency $N_{f}=1$ and choose $\theta=\pi / 20$ for the rotation angle of the square. The Störmer-Verlet method (A 30)-(A 33) conserves energy in time up to fluctuations of amplitude $O\left(\tau^{2}\right)$. For this choice of $\tau$ the relative error of the Hamiltonian function (3.7) remained smaller than $10^{-3}$ in all three numerical experiments. Computational results with initial conditions $\psi_{1,1}$ and $\psi_{1,3}$ are shown in 
(a)

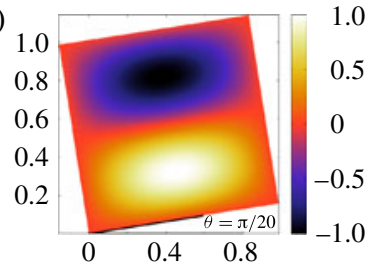

(b)

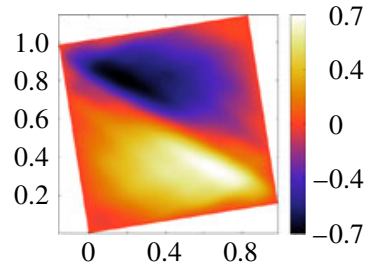

(c)

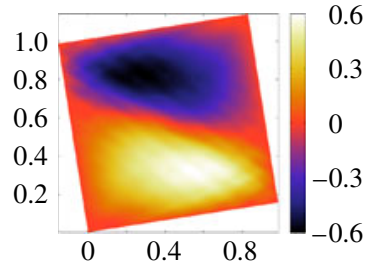

FIGURE 6. (Colour online) Evolution of the stream function in time from the initial condition $\psi_{1,2}:(a) \psi, t=0 ;(b) \psi, t=100 ;(c) \psi, t=200$.

figure 2. Results with the initial condition $\psi_{1,2}$ are shown in figure 6 . In all three examples we plot the evolution of the stream function at three distinct times.

Complementary to the state variables we also look at the energy density function, i.e. the distribution of the energy in space. Hence we define the discrete energy density function at the cell centres, making use of the discrete velocities defined by (A 17),

$$
E_{i+1 / 2, j+1 / 2}=\frac{1}{2} u_{i+1 / 2, j+1 / 2}^{2}+\frac{1}{2} w_{i+1 / 2, j+1 / 2}^{2}+\frac{1}{2 N_{f}^{2}} b_{i+1 / 2, j+1 / 2}^{2} .
$$

In the numerical example with initial condition $\psi_{1,1}$, we observe that energy that is initially concentrated at the low wavenumber is transported to large wavenumbers. Evidently, in figure 2 the whole family of $(1,1)$ attractors is observable. The evolution from initial condition $\psi_{1,3}$ is similar, but in this case the family of $(1,3)$ wave attractors is obtained: see figure 2 . On the other hand, with initial condition $\psi_{1,2}$ the solution appears to consist mainly of a strong periodic component, plus small-scale fluctuations.

We have seen that the energy functional (2.12) is conserved along the solution of the continuous system (2.8)-(2.11). Furthermore, backward error analysis of symplectic numerical integrators (Leimkuhler \& Reich 2004; Hairer et al. 2006) applied to Hamiltonian systems shows the existence of a perturbed Hamiltonian of the form $H+O\left(\tau^{2}\right)$ which is exactly conserved. For our problem, this implies the discrete total energy (3.7) will be conserved up to bounded fluctuations with amplitude $O\left(\tau^{2}\right)$ along the solution of the discrete system (A 30)-(A 33). Nevertheless, we observe that the amplitude of the stream function decays. That can be seen by comparing the intensity bars in figures 2 and 6. For total energy to remain constant, there should either be a net exchange of kinetic into potential energy, or the amplitude of vorticity should grow commensurate to the loss in stream function. To confirm this we study the time series of the state variables: stream function, vorticity, buoyancy, velocities (A 17) and the energy density function (4.2), at three arbitrarily chosen points in space. These three points are shown in figure 2(a). In figure 7 we plot numerical time series data at these three points for the initial condition $\psi_{1,1}$. From figure 7 we see that for energy to stay bounded when the amplitude of the stream function decays the amplitude of the vorticity grows and buoyancy, energy density function and the components of the velocity field stay bounded. This is reminiscent of the familiar cascade of vorticity to large wavenumbers in two-dimensional fluids, but note that the nonlinear advection terms are neglected in this model, so the observed effect is really due to dispersion among the normal modes.

The presence of only a single family of wave attractors in the time evolution of the initial conditions $\psi_{1,1}$ and $\psi_{1,3}$ suggests the excitation of only those frequencies 

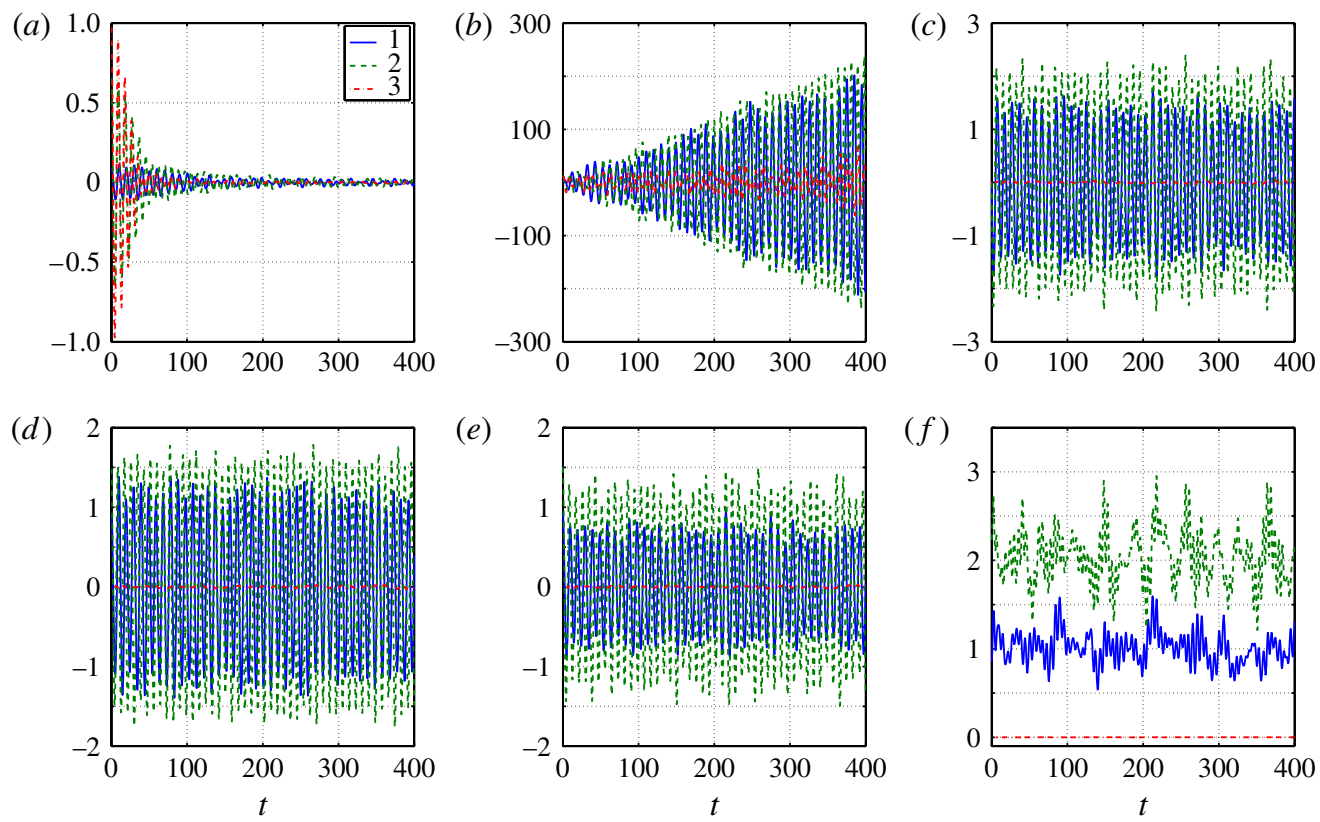

FIGURE 7. (Colour online) Time series of the stream function, vorticity, buoyancy, velocity $u$, velocity $w$ and energy density function $E$ at 3 points in space from computations with initial condition $\psi_{1,1}$ and $b=0:(a) \psi$ at 3 points; $(b) q$ at 3 points; $(c) b$ at 3 points; $(d) u$ at 3 points; $(e) w$ at 3 points; $(f) E$ at 3 points.
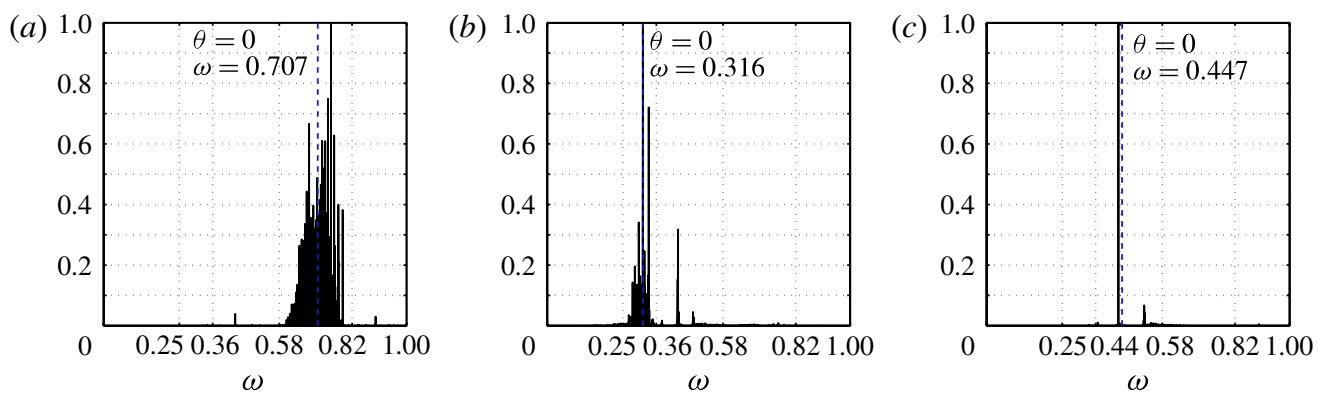

FIGURE 8. (Colour online) Energy projections upon normal modes of the semi-discrete system (3.4)-(3.5) for initial conditions: (a) $\psi_{1,1}, H_{i} / \max \left\{H_{i}\right\} ;(b) \psi_{1,3}, H_{i} / \max \left\{H_{i}\right\} ;(c)$ $\psi_{1,2}, H_{i} / \max \left\{H_{i}\right\}$.

associated to the respective class of $(1,1)$ and $(1,3)$ wave attractors, respectively. Similarly, the nearly periodic evolution from the $\psi_{1,2}$ Fourier mode suggests the dominance of the periodic $(1,2)$ solution.

To understand this, we project the Fourier modes onto the normal modes of the tilted system. We expand the initial conditions (4.1) in the normal modes of the semi-discretization (3.4)-(3.5) for $\theta=\pi / 20$ and $N_{f}=1$ and plot the scaled discrete energy values $H_{i} / \max \left\{H_{i}\right\}$ with respect to the frequencies of the discrete system in figure $8(a, b, c)$. In each of these figures we plot a dashed line to indicate the standing 


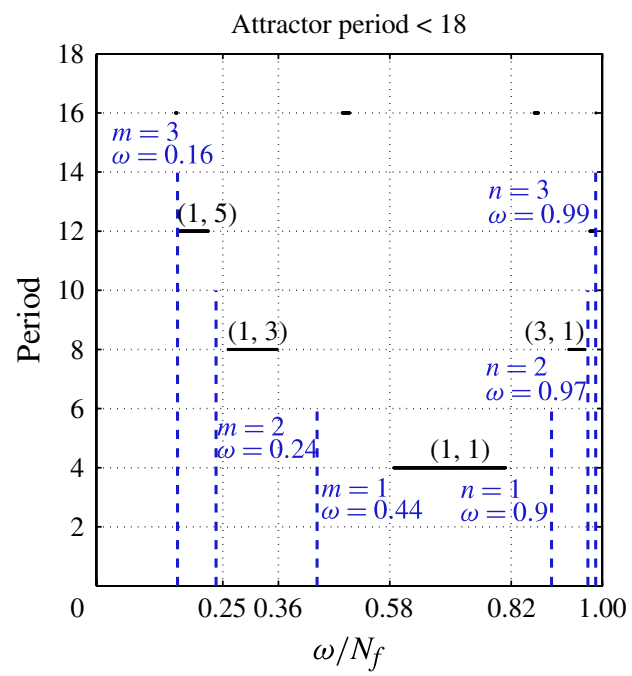

FIGURE 9. (Colour online) Attractor period as a function of subharmonic forcing frequency $\omega / N_{f}$ for the fixed angle $\theta=\pi / 20$. Horizontal bars indicate families of limit cycle attractors; dashed lines indicate the discrete periodic cases.

wave solution frequency for $\theta=0$. The data for figure 9 were taken from the crosssection of figure $3(b)$ corresponding to tilt angle $\theta=\pi / 20$, and were computed by following characteristics. The figure indicates the attractor periods of the limit cycles observed as a function of (subharmonic) forcing frequency, for attractors having period less than eighteen. The horizontal bars reflect the fact that there is a continuous range of forcing frequencies that lead to limit cycle attractors of a given geometry, e.g. the class of $(1,1)$ attractors having period 4 . For $\theta=\pi / 20$ there exist precisely six periodic solutions of type $(2 n, 1)$ and $(1,2 m)$ whose discrete frequencies are indicated by the vertical dashed lines. Comparing figures 9 and $8(a)$ we see that the $(1,1)$ Fourier mode projects almost entirely onto the range of $(1,1)$ attractors. Since there is no energy transfer between normal modes, the solution of the semi-discrete system with initial conditions $\psi_{1,1}$ at any time is a linear combination of the normal modes with frequencies in the range of the $(1,1)$ attractors. Similarly, most of the energy in the $(1,3)$ Fourier mode projects onto the range of $(1,3)$ attractors: figure $8(b)$. In contrast, figure $8(c)$ illustrates that the $(1,2)$ Fourier mode is concentrated at one discrete frequency, which is very near that of the $(1,2)$ periodic solution, explaining the nearly periodic behaviour of this solution.

For future reference, figure 10 shows normal modes with frequencies within the $(1,1)$ and $(1,3)$ attractor ranges, as well as the distinct normal mode with $(1,2)$ periodic solution frequency. The normal modes shown in figure 10 are those whose frequencies are closest to the forcing frequencies of the monochromatic solutions in figure 4. The same frequencies were used to generate the Floquet exponents plotted in figure $5(a, b)$, and to force the solutions shown in figure 11 . The normal modes displayed in figure $10(a, b)$ are irregular, with high-frequency oscillations near the grid scale, but a low-frequency plateau structure is also evident. We have inspected a number of the normal modes having frequencies in the $(1,1)$ and $(1,3)$ attractor regimes. A subset of these possess a large-scale structure in which attractor geometry is discernible, as with figure $10(a, b)$. On the other hand, many of the normal modes 
(a)

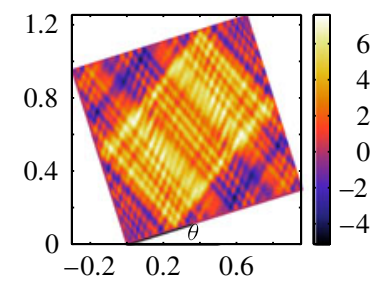

(b)

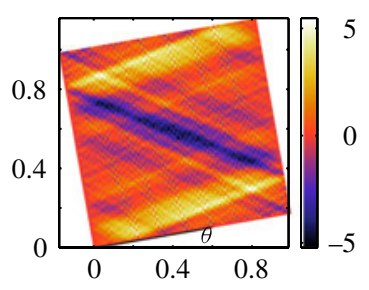

(c)

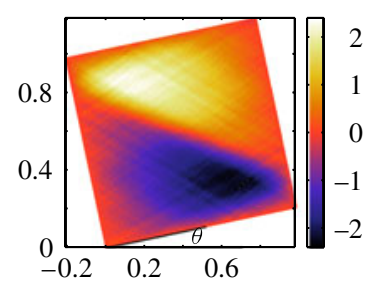

FIgURE 10. (Colour online) Normal modes of the stream function $\left(N_{f}=1\right)$ : $(a)$ within the $(1,1)$ attractor frequency range $(\omega=0.74, \theta=7 \pi / 72) ;(b)$ within the $(1,3)$ attractor frequency range $(\omega=0.34, \theta=\pi / 18) ;(c)$ the $(1,2)$ periodic solution $(\omega=0.43, \theta=\pi / 15)$.

(a)

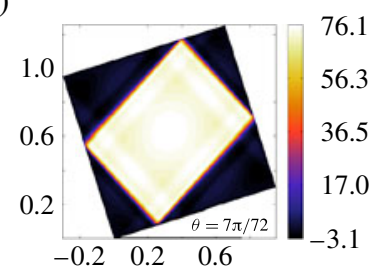

(d)

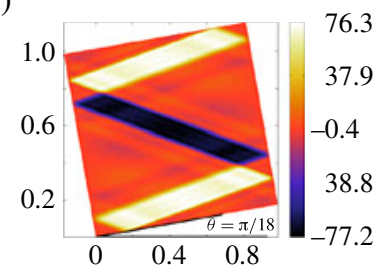

(b)

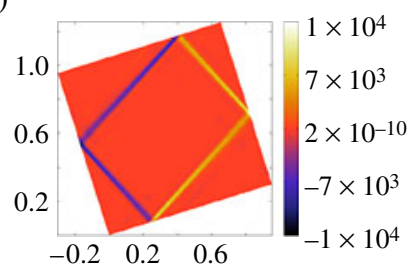

(e)

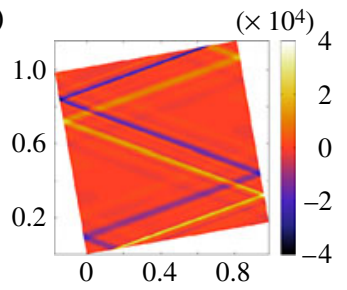

(c)

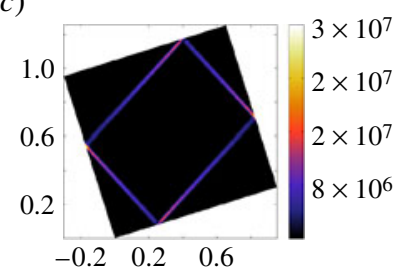

(f)

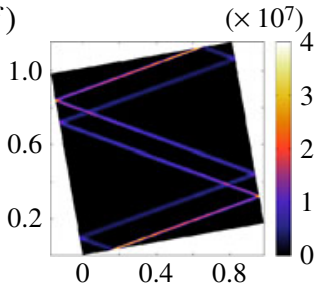

FIgURE 11. (Colour online) Wave attractors after 50 forcing periods (i.e. $t=200$ ): $(a-c)(1,1)$ attractor regime, and $(d-f)(1,3)$ attractor regime. $(a, d)$ Stream function, $(b, e)$ buoyancy, and $(c, f)$ energy density. The initial conditions are the same as in figure 2 at time $t=0$ : (a) $\psi, t=200 ;(b) b, t=200 ;(c) E, t=200 ;(d) \psi, t=200 ;(e) b, t=200$; (f) $E, t=200$.

have no apparent relation to the attractor structure. Furthermore, we were unable to see any functional relation between the normal-mode structure and either frequency or resolution. This is perhaps unsurprising, when one considers that these solutions form an orthogonal basis (in an appropriate inner product) for the discrete stream function space.

In summary, for the untilted case the response to an initial perturbation corresponds to an $(n, m)$ normal mode that simply 'sloshes' sinusoidally in time at the single frequency associated with that mode. In this case there are no other frequencies excited. When the same initial spatial perturbation is given in the tilted square domain, most of its energy is projected onto the whole ensemble of $(n, m)$ attractor modes, each associated with a different frequency residing in the $(n, m)$ frequency window. 


\subsection{Computation of wave attractors}

In $\S 2$ we described how to compute monochromatic wave solutions in a tilted square. We illustrated this with two examples of internal gravity wave attractors: see figure $4(a, b)$. In this section we compute internal wave attractors as an initial value problem with parametric excitation, so-called parametric resonance solutions.

We solve (3.4)-(3.5) with the Störmer-Verlet method. Since we generate instability in the system by parametric excitation, the amplitude of the solution grows in time, and energy is no longer conserved. We choose forcing frequency $2 \omega=\pi$ such that the wave period is $T=4$ and choose the normalized subharmonic frequency $\omega / N_{f}$ and tilt angle $\theta$ on the basis of the type of limit behaviour we want to simulate. We compute a $(1,1)$ attractor with parameter values $\omega / N_{f}=0.74$ and $\theta=7 \pi / 72$, and a $(1,3)$ attractor with parameter values $\omega / N_{f}=0.34$ and $\theta=\pi / 18$.

Numerical parameters are fixed for both experiments: the forcing amplitude $\epsilon=0.1$, time step $\tau=0.05$ and grid step sizes $\Delta x=\Delta z=2 \times 10^{-3}$. The initial conditions are chosen to be the Fourier modes $\psi_{1,1}$ and $\psi_{1,3}$ in the computation of the $(1,1)$ and $(1,3)$ attractors, respectively. We force the system for 50 wave periods and plot the stream function, buoyancy and the discrete energy density function (4.2) at the final time in figure 11.

Figure 11(a-c) displays the results for the $(1,1)$ limit cycle attractor. The energy is focused on the attractor, which reflects from each side of the square once. We observe a standing wave solution with growing amplitude and a 'plateau' type of attractor with piecewise constant stream function. After $\sim 10$ wave periods, i.e. at time $t=40$, the wave motion becomes localized along the straight lines of the attractor. The same 'plateau' type of attractors were observed in laboratory experiments (Hazewinkel et al. 2008). Since all sides of the tilted square are inclined, in the case of a simple $(1,1)$ attractor, internal waves become focused at all boundaries, because the energy is transported in an anticlockwise orientation around the attractor, as is indicated in the plots of the energy density function: see e.g. figure 11(c). (Note that due to focusing, the energy density increases after reflection. Hence, the anticlockwise direction of energy propagation on the attractor can be deduced from the energy density plots.)

In figure $11(d-f)$ we consider an example of a $(1,3)$ attractor. It has one reflection point with the upper and lower boundaries of the square, and three reflection points each on the left and right sides of the square. Similarly to the case of the $(1,1)$ attractor, we observe a standing wave solution that grows in amplitude, and the wave energy is localized along the straight lines of an attractor. The form of the attractor is again of 'plateau' type. Internal waves become highly focused upon reflection from the upper and lower boundaries of the square and gradually defocus in the rest of the domain: see figure $11(f)$.

Following the discussion of $\S 4.1$, the choice of the initial conditions $\psi_{1,1}$ and $\psi_{1,3}$ ensures that there will be significant energy in the normal modes corresponding to $(1,1)$ and $(1,3)$ attractors, a subset of which will grow in amplitude due to resonance of the underlying Mathieu equations. Those modes with frequencies outside the instability tongue of the Mathieu equations remain bounded for all times and eventually become negligible compared to the unstable modes. Since we do not have external damping (as in the experiment discussed in Maas et al. 1997 and Lam \& Maas 2008), these modes also do not dissipate. Evolution of the stable modes is primarily significant only during the early part of the simulation, before the wave attractor dominates.

Experiments with smaller values of $\epsilon$ result in increased focusing in the neighbourhood of the attractor. Figure 5 suggests that early on in the computation 

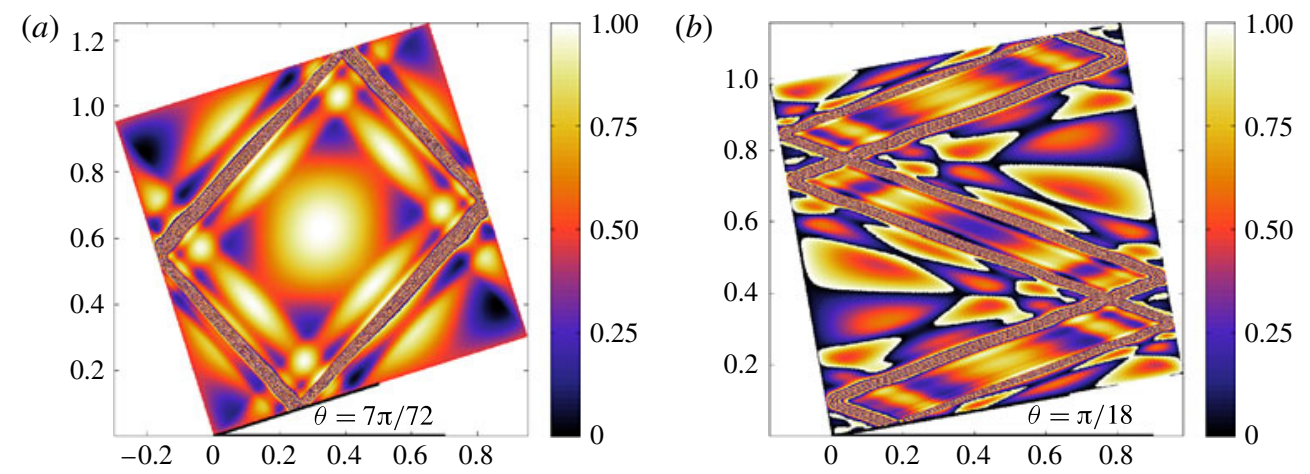

FIgURE 12. (Colour online) Deviation from a piecewise constant solution, after 50 wave periods: $(a)(1,1)$ attractor, small scales of $\psi, t=200 ;(b)(1,3)$ attractor, small scales of $\psi, t=200$.

all the normal modes with frequencies in the resonant zone contribute to the dynamics. But since those modes for which the real part of the Floquet exponent is greater grow much faster in time, these become more prominently visible than others. Because of this, energy becomes more and more focused near the attractor as time progresses.

Since there is no exchange of energy between normal modes, the precise structure observed at large times will depend both on the associated Floquet multipliers and on the initial distribution of energy among the resonant frequencies. In other words, the initial condition is relevant to what is observed in figure 11. On an intermediate time scale (here, 50 forcing periods), those normal modes whose frequencies are associated with the largest Floquet multipliers dominate the solution, and the observed steadily focusing attractor structure is a linear combination of these modes. If integration is carried out for much, much longer times (e.g. thousands of forcing periods for the current resolution), eventually only the distinct normal mode of the largest Floquet multiplier will be observable. This can largely be considered a numerical artifact, in many cases having no recognizable attractor pattern, nor corresponding to any physical solution. In the presence of viscosity, the various normal modes do not evolve independently (cf. equation (B 15) in appendix B), and the asymptotic solution is independent of the initial condition (Ogilvie 2005).

Typical normal modes are non-smooth, for example, as shown in figure 10. The solutions observed in figure 11 are primarily of plateau type. These solutions are composite, consisting of a linear combination of the most resonant modes. Close inspection of the solutions in figure 11 reveals that the plateaus are not perfectly flat, but that there are secondary oscillations of smaller amplitude present. To better observe these, we subtract the plateau solution using the following formula:

$$
\delta \psi_{i, j}=\operatorname{trunc}\left(\frac{\psi_{i, j}-\min \{\boldsymbol{\psi}\}}{\max \{\boldsymbol{\psi}-\min \{\boldsymbol{\psi}\}\}} k\right), \quad \operatorname{trunc}(f)=f-\lfloor f\rfloor,
$$

where $\lfloor f\rfloor$ indicates the largest integer less than $f$. The idea of the formula is to rescale the stream function, such that the oscillations about the plateau solution have an amplitude that is less than unity, and then subtract the integer part of the solution everywhere. This is achieved for the empirically chosen value $k=12$. We plot the secondary wave solution in figure 12 for the stream function at final time $t=200$. Note the symmetry of the solution and a passing resemblance to figure 4 , for 
which half-cosine waves were prescribed on the fundamental intervals. The secondary solutions are also robust with respect to spatial resolution and time step $\tau$. The shape of the secondary solution and its robustness with respect to numerical parameters and perturbation amplitude $\varepsilon$ suggests that the attractor shape is not truly piecewise constant, but has higher-order secondary waveforms.

\section{Conclusions}

In this paper we have considered the simplest time-dependent configuration in which internal wave attractors can be generated in stratified fluids: linearized, inviscid flow with parametric forcing. We constructed a symmetric, energy-conserving finite difference method. For the case of a tilted square geometry we simulated both the free evolution (unforced) wave evolution from Fourier mode initial conditions, and the parametrically forced evolution towards a wave attractor. This simple configuration, as well as the symmetries of the discretization, permit a complete normal-mode analysis of the initial value problem in the discrete case. Based on this analysis we can conclude that the finite-dimensional approximation has a complete basis of normal modes that is invariant in time, meaning that the initial value problem can be fully decoupled into scalar harmonic oscillators, each of which preserves its initial energy. Therefore, the numerical solution is quasi-periodic, although the Poincare recurrence time (the time over which a discrete, energy-conserving system recovers its initial state) may be quite large. The same analysis can be carried out for the parametrically forced case, showing that the forced system of ordinary differential equations (ODEs) can be completely decoupled into Mathieu equations. For a generic initial condition, and depending on the frequency and magnitude of forcing, a range of normal-mode frequencies will lie in an Arnold tongue of instability, and the corresponding modes will grow in time, eventually dominating the solution and forming a wave attractor. The shape of the stream function is to first order a plateau, or a piecewise constant function, but there are secondary solutions that are robust with respect to discretization and forcing parameters.

We remark that for a given forcing, it is possible to choose judiciously an initial condition whose projection onto the amplified frequencies of the Mathieu equation is zero. In this case, a wave attractor will never be generated. However, this no longer holds if nonlinear advection is taken into account, due to nonlinear coupling. In fact, even for the linearized model, if viscosity is included there is no global decomposition into scalar dynamics, since the normal-mode decomposition becomes time-dependent.

\section{Acknowledgement}

This research was partly financed by a grant from the Netherlands Research Council NWO.

\section{Appendix A. Hamiltonian numerical discretization}

The Euler equations for an ideal fluid have a well-known Hamiltonian structure (Arnold 1989; Morrison 1998) that strongly constrains the dynamics. When constructing approximate models such as the Euler-Boussinesq equations (2.3)-(2.6), it is usually advisable to preserve this structure (Salmon 1998). As shown in Holm, Marsden \& Ratiu (2002), the nonlinear Euler-Boussinesq equations inherit the noncanonical Hamiltonian structure from the ideal fluid Poisson bracket. Here we verify that the linearization leading to (2.8)-(2.11) also preserves a linear Hamiltonian 
structure. A system of partial differential equations (PDEs) on a function space $\boldsymbol{F}^{d}$ equipped with an inner product $(\cdot, \cdot): \boldsymbol{F}^{d} \times \boldsymbol{F}^{d} \rightarrow \mathbb{R}$ is said to constitute a Hamiltonian system (Olver 1986) in the variables $\boldsymbol{f}(x, t)=\left(f_{1}(x, t), \ldots, f_{d}(x, t)\right)^{\mathrm{T}} \in \boldsymbol{F}^{d}$ if there exists a functional $\mathscr{H}(\boldsymbol{f}): \boldsymbol{F}^{d} \rightarrow \mathbb{R}$ and a constant, $d \times d$ matrix differential operator (structure matrix) $\mathscr{J}: \boldsymbol{F}^{d} \rightarrow \boldsymbol{F}^{d}$, that is skew-symmetric with respect to $(\cdot, \cdot)$, such that the PDE can be expressed as

$$
\partial_{t} \boldsymbol{f}=\mathscr{J} \frac{\delta \mathscr{H}}{\delta \boldsymbol{f}}
$$

where the variational derivative $\delta \mathscr{H} / \delta \boldsymbol{f}$ is defined by

$$
\left(\frac{\delta \mathscr{H}}{\delta \boldsymbol{f}}, \boldsymbol{g}\right)=\lim _{\varepsilon \rightarrow 0} \frac{1}{\varepsilon}[\mathscr{H}(\boldsymbol{f}+\varepsilon \boldsymbol{g})-\mathscr{H}(\boldsymbol{f})], \quad \forall \boldsymbol{g} \in \boldsymbol{F}^{d} .
$$

One consequence of Hamiltonian structure is the conservation of the Hamiltonian along solutions of (A 1), which follows from

$$
\frac{d \mathscr{H}}{d t}=\left(\frac{\delta \mathscr{H}}{\delta \boldsymbol{f}}, \partial_{t} \boldsymbol{f}\right)=\left(\frac{\delta \mathscr{H}}{\delta \boldsymbol{f}}, \mathscr{J} \frac{\delta \mathscr{H}}{\delta \boldsymbol{f}}\right)=0
$$

by the skew-symmetry condition on $\mathscr{J}$.

We show the following.

Proposition 1. For any value of $\theta$ the linearized Euler-Boussinesq equations in the stream function formulation (2.8)-(2.11) can be written as a non-canonical Hamiltonian system $(A 1)$ in the $L^{2}$ inner product with $f=(q, b)$, structure matrix

$$
\mathscr{J}=-N_{f}^{2} \cos \theta\left[\begin{array}{cc}
0 & \partial_{x} \\
\partial_{x} & 0
\end{array}\right]+N_{f}^{2} \sin \theta\left[\begin{array}{cc}
0 & \partial_{z} \\
\partial_{z} & 0
\end{array}\right]
$$

and Hamiltonian

$$
\mathscr{H}=\frac{1}{2} \int_{D}\left(\nabla \psi \cdot \nabla \psi+\frac{1}{N_{f}^{2}} b^{2}\right) \mathrm{d} \boldsymbol{x}
$$

Proof. The first variations of the Hamiltonian functional (A 5) with respect to $q$ and $b$ are

$$
\begin{aligned}
\delta \mathscr{H} & =\int_{D}\left(\nabla \psi \cdot \nabla \delta \psi+\frac{1}{N_{f}^{2}} b \delta b\right) \mathrm{d} \boldsymbol{x} \\
& =\int_{D}\left(-\psi \Delta \delta \psi+\frac{1}{N_{f}^{2}} b \delta b\right) \mathrm{d} \boldsymbol{x}=\int_{D}\left(\psi \delta q+\frac{1}{N_{f}^{2}} b \delta b\right) \mathrm{d} \boldsymbol{x},
\end{aligned}
$$

where the boundary condition (2.11) has been used to carry out the integration by parts. It follows that the variational derivatives of the Hamiltonian (A 5) with respect to the vorticity $q$ and the buoyancy $b$ are

$$
\frac{\delta \mathscr{H}}{\delta q}=\psi, \quad \frac{\delta \mathscr{H}}{\delta b}=\frac{1}{N_{f}^{2}} b
$$


Substituting (A 7) and (A 4) into (A 1), we get that

$$
\mathscr{J} \frac{\delta \mathscr{H}}{\delta \boldsymbol{f}}=\mathscr{J}\left(\begin{array}{c}
\frac{\delta \mathscr{H}}{\delta q} \\
\frac{\delta \mathscr{H}}{\delta b}
\end{array}\right)=\left(\begin{array}{c}
-\partial_{x} b \cos \theta+\partial_{z} b \sin \theta \\
-N_{f}^{2}\left(\partial_{x} \psi \cos \theta-\partial_{z} \psi \sin \theta\right)
\end{array}\right)=\left(\begin{array}{c}
\partial_{t} q \\
\partial_{t} b
\end{array}\right)=\partial_{t} f
$$

which agrees with (2.8)-(2.11).

It follows that the Hamiltonian functional (A 5) is conserved along the solution of the equation system (2.8)-(2.11).

\section{A.1. Finite difference matrices}

In this section we describe a numerical discretization for the Euler-Boussinesq equations that preserves a discrete analogue of the Hamiltonian structure in the inviscid, unforced limit. In particular, the spatially discrete system of ODEs has a first integral approximating the energy. The scheme also preserves the symmetries of the continuous differential operators. Our approach is to discretize the Hamiltonian and structure operator $\mathscr{J}$ separately, while enforcing the skew-symmetry of $\mathscr{J}$ (see McLachlan 1995). Although this approach leads to a rather standard staggered central difference scheme here, it can be used to construct a Hamiltonian discretization on more general domains and non-uniform grids, which will be important for studying internal waves in ocean basins.

Consider the unit square domain $D=[0,1]^{2}$ divided into $N_{x} \times N_{z}$ uniform rectangular cells. Subscripted indices indicate grid nodes $\boldsymbol{x}_{i, j}=(i \Delta x, j \Delta z)$, where $\Delta x=1 / N_{x}$ and $\Delta z=1 / N_{z}$ are the grid sizes in the $x$ and $z$ directions, respectively. We shall construct a Hamiltonian structure-preserving staggered finite difference scheme. To this end let us denote by $\boldsymbol{C}=\mathbb{R}^{N_{x} \times N_{z}}$ the space of cell-centred grid functions and by $\boldsymbol{V}=\mathbb{R}^{\left(N_{x}-1\right) \times\left(N_{z}-1\right)}$ the space of grid functions defined at cell vertices, where in the latter case we only include inner vertices, since the boundary vertices are either known or not needed in the discretization.

The discrete stream function $\psi_{i, j}$ and vorticity $q_{i, j}$ are defined at cell vertices and the buoyancy $b_{i+1 / 2, j+1 / 2}$ at cell centres. The discrete analogue of the boundary condition on the stream function $(2.11)$ is

$$
\psi_{0, j}=\psi_{N_{x}, j}=0, \quad \forall j, \quad \psi_{i, 0}=\psi_{i, N_{z}}=0, \quad \forall i .
$$

We define column vectors $\boldsymbol{q}, \boldsymbol{\psi} \in \boldsymbol{V}$ consisting only of the interior grid point values of $q_{i, j}$ and $\psi_{i, j}$. The buoyancy column vector $\boldsymbol{b} \in \boldsymbol{C}$ consists of all the values of $b_{i+1 / 2, j+1 / 2}$ defined at cell centres.

We also define discrete inner products on $\boldsymbol{C}$ and $\boldsymbol{V}$ :

$$
\begin{gathered}
\langle\boldsymbol{a}, \boldsymbol{b}\rangle_{\boldsymbol{C}}=\sum_{i, j=0}^{N_{x}-1, N_{z}-1} a_{i+1 / 2, j+1 / 2} b_{i+1 / 2, j+1 / 2} \Delta x \Delta z, \quad \boldsymbol{a}, \boldsymbol{b} \in \boldsymbol{C}, \\
\langle\boldsymbol{q}, \boldsymbol{r}\rangle_{\boldsymbol{V}}=\sum_{i, j=1}^{N_{x}-1, N_{z}-1} q_{i, j} r_{i, j} \Delta x \Delta z, \quad \boldsymbol{q}, \boldsymbol{r} \in \boldsymbol{V} .
\end{gathered}
$$

For the inner product on $\boldsymbol{V}$ we assume zero boundary data for at least one of its arguments.

Taking into account the discrete boundary conditions (A 9), the following matrices implement the central finite difference approximations to the first derivatives on 
cell edges:

$$
\left(\boldsymbol{D}_{x} \boldsymbol{\psi}\right)_{i+1 / 2, j}=\frac{\psi_{i+1, j}-\psi_{i, j}}{\Delta x}, \quad\left(\boldsymbol{D}_{z} \boldsymbol{\psi}\right)_{i, j+1 / 2}=\frac{\psi_{i, j+1}-\psi_{i, j}}{\Delta z},
$$

where $\boldsymbol{D}_{x} \in \mathbb{R}^{N_{x}\left(N_{z}-1\right) \times\left(N_{x}-1\right)\left(N_{z}-1\right)}$ and $\boldsymbol{D}_{z} \in \mathbb{R}^{N_{z}\left(N_{x}-1\right) \times\left(N_{x}-1\right)\left(N_{z}-1\right)}$. The dual operators $-\boldsymbol{D}_{x}^{\mathrm{T}}$ and $-\boldsymbol{D}_{z}^{\mathrm{T}}$ represent central finite difference approximations to the first derivatives on cell vertices from cell edges.

Further, we define the averaged operator matrices $\boldsymbol{M}_{x} \in \mathbb{R}^{N_{z}\left(N_{x}-1\right) \times N_{x} N_{z}}$ and $\boldsymbol{M}_{z} \in$ $\mathbb{R}^{N_{x}\left(N_{z}-1\right) \times N_{x} N_{z}}$ from cell centres to cell edges such that

$$
\left(\boldsymbol{M}_{x} \boldsymbol{b}\right)_{i, j+1 / 2}=\frac{b_{i+1 / 2, j+1 / 2}+b_{i-1 / 2, j+1 / 2}}{2}, \quad\left(\boldsymbol{M}_{z} \boldsymbol{b}\right)_{i+1 / 2, j}=\frac{b_{i+1 / 2, j+1 / 2}+b_{i+1 / 2, j-1 / 2}}{2},
$$

where their transposes are averaged operator matrices from the cell edges to the cell centres.

The matrices above can be composed in various ways to construct approximate derivative operators from $\boldsymbol{V}$ to $\boldsymbol{C}$ and vice versa:

$$
\boldsymbol{M}_{z}^{\mathrm{T}} \boldsymbol{D}_{x}: \boldsymbol{V} \rightarrow \boldsymbol{C}, \quad \boldsymbol{M}_{x}^{\mathrm{T}} \boldsymbol{D}_{z}: \boldsymbol{V} \rightarrow \boldsymbol{C}, \quad-\boldsymbol{D}_{x}^{\mathrm{T}} \boldsymbol{M}_{z}: \boldsymbol{C} \rightarrow \boldsymbol{V}, \quad-\boldsymbol{D}_{z}^{\mathrm{T}} \boldsymbol{M}_{x}: \boldsymbol{C} \rightarrow \boldsymbol{V} .
$$

The discrete Laplacian operator $L: V \rightarrow \boldsymbol{V}$, defined by

$$
\boldsymbol{L}=-\left(\boldsymbol{D}_{x}^{\mathrm{T}} \boldsymbol{D}_{x}+\boldsymbol{D}_{z}^{\mathrm{T}} \boldsymbol{D}_{z}\right) \in \mathbb{R}^{\left(N_{x}-1\right)\left(N_{z}-1\right) \times\left(N_{x}-1\right)\left(N_{z}-1\right)},
$$

is the standard symmetric, negative definite, five-point central difference stencil, i.e.

$$
(\boldsymbol{L} \psi)_{i, j}=\frac{\psi_{i+1, j}-2 \psi_{i, j}+\psi_{i-1, j}}{\Delta x^{2}}+\frac{\psi_{i, j+1}-2 \psi_{i, j}+\psi_{i, j-1}}{\Delta z^{2}},
$$

where the boundary terms are modified to satisfy (A 9). We define the discrete vorticity field by $q=-L \psi$.

For diagnostic purposes we also define the discrete velocity components at cell centres:

$$
\boldsymbol{u}=-\boldsymbol{M}_{x}^{\mathrm{T}} \boldsymbol{D}_{z} \boldsymbol{\psi}, \quad \boldsymbol{w}=\boldsymbol{M}_{z}^{\mathrm{T}} \boldsymbol{D}_{x} \boldsymbol{\psi} .
$$

\section{A.2. Hamiltonian semi-discretization}

To construct a Hamiltonian semi-discretization with structure analogous to (A4), we define a quadrature for $H$ and a skew-symmetric structure that approximates $\mathscr{J}$.

In terms of inner products on $\boldsymbol{C}$ and $\boldsymbol{V}$, the discrete Hamiltonian is defined by

$$
H(\boldsymbol{q}, \boldsymbol{b})=\frac{1}{2}\left(-\langle\boldsymbol{\psi}, \boldsymbol{q}\rangle_{V}+\frac{1}{N_{f}^{2}}\langle\boldsymbol{b}, \boldsymbol{b}\rangle_{C}\right)=\frac{1}{2}\left(-\left\langle\boldsymbol{q}, \boldsymbol{L}^{-1} \boldsymbol{q}\right\rangle_{V}+\frac{1}{N_{f}^{2}}\langle\boldsymbol{b}, \boldsymbol{b}\rangle_{C}\right) .
$$

The variational derivatives of $H$ are defined in the weak sense in these inner products by

$$
\begin{gathered}
\left\langle\frac{\delta H}{\delta \boldsymbol{q}}, \boldsymbol{r}\right\rangle_{V}=\lim _{\varepsilon \rightarrow 0} \frac{1}{\varepsilon}(H(\boldsymbol{q}+\varepsilon \boldsymbol{r}, \boldsymbol{b})-H(\boldsymbol{q}, \boldsymbol{b}))=\langle\boldsymbol{\psi}, \boldsymbol{r}\rangle_{V}, \quad \forall \boldsymbol{r} \in \boldsymbol{V}, \\
\left\langle\frac{\delta H}{\delta \boldsymbol{b}}, \boldsymbol{a}\right\rangle_{\boldsymbol{C}}=\lim _{\varepsilon \rightarrow 0} \frac{1}{\varepsilon}(H(\boldsymbol{q}, \boldsymbol{b}+\varepsilon \boldsymbol{a})-H(\boldsymbol{q}, \boldsymbol{b}))=\left\langle\frac{1}{N_{f}^{2}} \boldsymbol{b}, \boldsymbol{a}\right\rangle_{\boldsymbol{C}}, \quad \forall \boldsymbol{a} \in \boldsymbol{C},
\end{gathered}
$$

i.e.

$$
\frac{\delta H}{\delta \boldsymbol{q}}=\boldsymbol{\psi}, \quad \frac{\delta H}{\delta \boldsymbol{b}}=\frac{1}{N_{f}^{2}} \boldsymbol{b}
$$


Next, we define a composite space $\boldsymbol{G}=\boldsymbol{V} \times \boldsymbol{C}$. A vector $\boldsymbol{g} \in \boldsymbol{G}$ takes the form

$$
\boldsymbol{g}=\left(\begin{array}{l}
g_{V} \\
g_{C}
\end{array}\right)
$$

where $\boldsymbol{g}_{\boldsymbol{V}} \in \boldsymbol{V}$ and $\boldsymbol{g}_{\boldsymbol{C}} \in \boldsymbol{C}$. We also define a joint inner product on $\boldsymbol{G}$,

$$
\langle\langle\boldsymbol{g}, \boldsymbol{h}\rangle\rangle=\left\langle\boldsymbol{g}_{V}, \boldsymbol{h}_{V}\right\rangle_{V}+\left\langle\boldsymbol{g}_{\boldsymbol{C}}, \boldsymbol{h}_{\boldsymbol{C}}\right\rangle_{\boldsymbol{C}},
$$

and the variational derivative

$$
\frac{\delta H}{\delta \boldsymbol{g}}=\left(\begin{array}{c}
\frac{\delta H}{\delta \boldsymbol{g}_{V}} \\
\frac{\delta H}{\delta \boldsymbol{g}_{C}}
\end{array}\right) .
$$

We approximate the structure operator (A 4) using our finite difference matrices:

$$
\boldsymbol{J}=-N_{f}^{2} \cos \theta\left[\begin{array}{cc}
0 & -\boldsymbol{D}_{x}^{\mathrm{T}} \boldsymbol{M}_{z} \\
\boldsymbol{M}_{z}^{\mathrm{T}} \boldsymbol{D}_{x} & 0
\end{array}\right]+N_{f}^{2} \sin \theta\left[\begin{array}{cc}
0 & -\boldsymbol{D}_{z}^{\mathrm{T}} \boldsymbol{M}_{x} \\
\boldsymbol{M}_{x}^{\mathrm{T}} \boldsymbol{D}_{z} & 0
\end{array}\right] .
$$

Note that $\boldsymbol{J}$ is skew-symmetric with respect to $\langle\cdot \cdot, \cdot\rangle\rangle$.

Choosing $\boldsymbol{g}=(\boldsymbol{q}, \boldsymbol{b})$, the Hamiltonian semi-discretization of the Euler-Boussinesq equations can now be defined by

$$
\frac{\mathrm{d} g}{\mathrm{~d} t}=\boldsymbol{J} \frac{\delta H}{\delta \boldsymbol{g}},
$$

or, in terms of $\boldsymbol{q}, \boldsymbol{b}$ and $\boldsymbol{\psi}$,

$$
\begin{aligned}
\frac{\mathrm{d} \boldsymbol{q}}{\mathrm{d} t} & =\boldsymbol{D}_{x}^{\mathrm{T}} \boldsymbol{M}_{z} \boldsymbol{b} \cos \theta-\boldsymbol{D}_{z}^{\mathrm{T}} \boldsymbol{M}_{x} \boldsymbol{b} \sin \theta, \\
\frac{\mathrm{d} \boldsymbol{b}}{\mathrm{d} t} & =-N_{f}^{2}\left(\boldsymbol{M}_{z}^{\mathrm{T}} \boldsymbol{D}_{x} \boldsymbol{\psi} \cos \theta-\boldsymbol{M}_{x}^{\mathrm{T}} \boldsymbol{D}_{z} \boldsymbol{\psi} \sin \theta\right), \\
\boldsymbol{q} & =-\boldsymbol{L} \boldsymbol{\psi} .
\end{aligned}
$$

By construction the discrete total energy $H$ is a first integral of the semidiscretization. Moreover, this system of ODEs is reversible and symplectic.

\section{A.3. Time integration}

We have shown that semi-discrete Euler-Boussinesq equations (A 27)-(A 29) constitute a time-reversible Hamiltonian system. We solve the Hamiltonian system in time with the symmetric and symplectic Störmer-Verlet method (Leimkuhler \& Reich 2004; Hairer et al. 2006),

$$
\begin{aligned}
\boldsymbol{q}^{n+1 / 2} & =\boldsymbol{q}^{n}+\frac{\tau}{2}\left(\boldsymbol{D}_{x}^{\mathrm{T}} \boldsymbol{M}_{z} \boldsymbol{b}^{n} \cos \theta-\boldsymbol{D}_{z}^{\mathrm{T}} \boldsymbol{M}_{x} \boldsymbol{b}^{n} \sin \theta\right), \\
\boldsymbol{\psi}^{n+1 / 2} & =-\boldsymbol{L}^{-1} \boldsymbol{q}^{n+1 / 2}, \\
\boldsymbol{b}^{n+1} & =\boldsymbol{b}^{n}-\tau N_{f}^{2}\left(\boldsymbol{M}_{z}^{\mathrm{T}} \boldsymbol{D}_{x} \boldsymbol{\psi}^{n+1 / 2} \cos \theta-\boldsymbol{M}_{x}^{\mathrm{T}} \boldsymbol{D}_{z} \boldsymbol{\psi}^{n+1 / 2} \sin \theta\right), \\
\boldsymbol{q}^{n+1} & =\boldsymbol{q}^{n+1 / 2}+\frac{\tau}{2}\left(\boldsymbol{D}_{x}^{\mathrm{T}} \boldsymbol{M}_{z} \boldsymbol{b}^{n+1} \cos \theta-\boldsymbol{D}_{z}^{\mathrm{T}} \boldsymbol{M}_{x} \boldsymbol{b}^{n+1} \sin \theta\right),
\end{aligned}
$$

such that the Hamiltonian function (A 18) will be conserved in time up to small fluctuations of second-order amplitude. The method requires the solution of the 
Poisson equation once per time step, but is otherwise explicit. We solve the Poisson equation efficiently using a fast Poisson solver. The overall method is second-order in space and time. Sparse discretization in space combined with a fast Poisson solver allows us to compute efficiently at high spatial resolution.

\section{Appendix B. Normal-mode decomposition of discretized linear internal waves}

We next consider the discrete model (3.4)-(3.5) with parametric forcing, written in terms of the stream function $\boldsymbol{\psi} \in \mathbb{R}^{M}$ and buoyancy $\boldsymbol{b} \in \mathbb{R}^{N}$ :

$$
\left[\begin{array}{cc}
-\boldsymbol{L} & 0 \\
0 & \frac{1}{N_{f}^{2}} \boldsymbol{I}_{N}
\end{array}\right] \frac{\mathrm{d}}{\mathrm{d} t}\left(\begin{array}{l}
\boldsymbol{\psi} \\
\boldsymbol{b}
\end{array}\right)=\left[\begin{array}{cc}
0 & \alpha(t) \boldsymbol{K} \\
-\boldsymbol{K}^{\mathrm{T}} & 0
\end{array}\right]\left(\begin{array}{l}
\boldsymbol{\psi} \\
\boldsymbol{b}
\end{array}\right),
$$

where $\boldsymbol{N}=\boldsymbol{N}_{x} \boldsymbol{N}_{z}, \boldsymbol{M}=\left(\boldsymbol{N}_{x}-1\right)\left(\boldsymbol{N}_{z}-1\right), \boldsymbol{L} \in \mathbb{R}^{M \times M}$ is the discrete approximation of the Laplacian (A 15), $\boldsymbol{K} \in \mathbb{R}^{M \times N}$ is a finite difference matrix

$$
\boldsymbol{K}=\boldsymbol{D}_{x}^{\mathrm{T}} \boldsymbol{M}_{z} \cos \theta-\boldsymbol{D}_{z}^{\mathrm{T}} \boldsymbol{M}_{x} \sin \theta,
$$

and $\boldsymbol{I}_{N}$ denotes the identity matrix on $\mathbb{R}^{N}$. The matrix $\boldsymbol{L}$ is symmetric and negative definite, and hence possesses an orthogonal basis of eigenvectors, and we can write $-\boldsymbol{L}=\boldsymbol{Q} \boldsymbol{D}_{\boldsymbol{L}} \boldsymbol{Q}^{\mathrm{T}}$, where $\boldsymbol{Q}^{\mathrm{T}} \boldsymbol{Q}=\boldsymbol{Q} \boldsymbol{Q}^{\mathrm{T}}=\boldsymbol{I}_{M}, \boldsymbol{Q} \in \mathbb{R}^{M \times M}$ and $\boldsymbol{D}_{\boldsymbol{L}} \in \mathbb{R}^{M \times M}$ is a diagonal matrix with positive entries. In matrix form we write

$$
\left[\begin{array}{cc}
\boldsymbol{Q} \boldsymbol{D}_{L} \boldsymbol{Q}^{\mathrm{T}} & 0 \\
0 & \frac{1}{N_{f}^{2}} \boldsymbol{I}_{N}
\end{array}\right] \frac{\mathrm{d}}{\mathrm{d} t}\left(\begin{array}{l}
\boldsymbol{\psi} \\
\boldsymbol{b}
\end{array}\right)=\left[\begin{array}{cc}
0 & \alpha(t) \boldsymbol{K} \\
-\boldsymbol{K}^{\mathrm{T}} & 0
\end{array}\right]\left(\begin{array}{l}
\boldsymbol{\psi} \\
\boldsymbol{b}
\end{array}\right)
$$

We transform as follows,

$$
\begin{aligned}
& {\left[\begin{array}{cc}
\boldsymbol{Q} \boldsymbol{D}_{\mathrm{L}}^{1 / 2} & 0 \\
0 & \frac{1}{N_{f}} \boldsymbol{I}_{N}
\end{array}\right]\left[\begin{array}{cc}
\boldsymbol{D}_{\mathrm{L}}^{1 / 2} \boldsymbol{Q}^{\mathrm{T}} & 0 \\
0 & \frac{1}{N_{f}} \boldsymbol{I}_{N}
\end{array}\right] \frac{\mathrm{d}}{\mathrm{d} t}\left(\begin{array}{l}
\boldsymbol{\psi} \\
\boldsymbol{b}
\end{array}\right)} \\
& =\left[\begin{array}{cc}
0 & \alpha(t) \boldsymbol{K} \\
-\boldsymbol{K}^{\mathrm{T}} & 0
\end{array}\right]\left[\begin{array}{cc}
\boldsymbol{Q} \boldsymbol{D}_{L}^{-1 / 2} & 0 \\
0 & N_{f} \boldsymbol{I}_{N}
\end{array}\right]\left[\begin{array}{cc}
\boldsymbol{D}_{\llcorner}^{1 / 2} \boldsymbol{Q}^{\mathrm{T}} & 0 \\
0 & \frac{1}{N_{f}} \boldsymbol{I}_{N}
\end{array}\right]\left(\begin{array}{l}
\boldsymbol{\psi} \\
\boldsymbol{b}
\end{array}\right),
\end{aligned}
$$

or, defining $\hat{\boldsymbol{\psi}}=\boldsymbol{D}_{L}^{1 / 2} \boldsymbol{Q}^{\mathrm{T}} \boldsymbol{\psi}$ and $\hat{\boldsymbol{b}}=\boldsymbol{b} / N_{f}$,

$$
\frac{\mathrm{d}}{\mathrm{d} t}\left(\begin{array}{l}
\hat{\boldsymbol{\psi}} \\
\hat{\boldsymbol{b}}
\end{array}\right)=N_{f}\left[\begin{array}{cc}
0 & \alpha(t) \boldsymbol{D}_{\boldsymbol{L}}^{-1 / 2} \boldsymbol{Q}^{\mathrm{T}} \boldsymbol{K} \\
-\boldsymbol{K}^{\mathrm{T}} \boldsymbol{Q} \boldsymbol{D}_{L}^{-1 / 2} & 0
\end{array}\right]\left(\begin{array}{l}
\hat{\boldsymbol{\psi}} \\
\hat{\boldsymbol{b}}
\end{array}\right) .
$$

Now let $\boldsymbol{C}=N_{f} \boldsymbol{D}_{L}^{-1 / 2} \boldsymbol{Q}^{\mathrm{T}} \boldsymbol{K} \in \mathbb{R}^{M \times N}$. The singular value decomposition of the real matrix $\boldsymbol{C}$ is denoted by

$$
\boldsymbol{C}=\boldsymbol{S} \Omega \boldsymbol{R}^{\mathrm{T}}
$$

where $\boldsymbol{S} \in \mathbb{R}^{M \times M}$ and $\boldsymbol{R} \in \mathbb{R}^{N \times N}$ are orthogonal matrices and $\boldsymbol{\Omega}=\operatorname{diag}\left(\omega_{1}, \ldots, \omega_{M}\right)$ is an $\mathbb{R}^{M \times N}$ matrix whose off-diagonals are zero and whose diagonal contains the $M$ real, positive singular values of $\boldsymbol{C}$. Hence (B 5) can be written as

$$
\frac{\mathrm{d}}{\mathrm{d} t}\left(\begin{array}{l}
\hat{\boldsymbol{\psi}} \\
\hat{\boldsymbol{b}}
\end{array}\right)=\left[\begin{array}{cc}
0 & \alpha(t) \boldsymbol{S} \boldsymbol{\Omega} \boldsymbol{R}^{\mathrm{T}} \\
-\boldsymbol{R} \boldsymbol{\Omega}^{\mathrm{T}} \boldsymbol{S}^{\mathrm{T}} & 0
\end{array}\right]\left(\begin{array}{l}
\hat{\boldsymbol{\psi}} \\
\hat{\boldsymbol{b}}
\end{array}\right) .
$$


Transforming again with $\tilde{\boldsymbol{\psi}}=\boldsymbol{S}^{\mathrm{T}} \hat{\boldsymbol{\psi}}$ and $\tilde{\boldsymbol{b}}=\boldsymbol{R}^{\mathrm{T}} \hat{\boldsymbol{b}}$ yields the system of (forced) harmonic oscillators

$$
\frac{\mathrm{d}}{\mathrm{d} t}\left(\begin{array}{l}
\tilde{\boldsymbol{\psi}} \\
\tilde{\boldsymbol{b}}
\end{array}\right)=\left[\begin{array}{cc}
0 & \alpha(t) \boldsymbol{\Omega} \\
-\boldsymbol{\Omega}^{\mathrm{T}} & 0
\end{array}\right]\left(\begin{array}{l}
\tilde{\boldsymbol{\psi}} \\
\tilde{\boldsymbol{b}}
\end{array}\right) .
$$

Expressed in terms of components, the above system becomes

$$
\begin{aligned}
\frac{\mathrm{d}^{2}}{\mathrm{~d} t^{2}} \tilde{\psi}_{i} & =-\alpha(t) \omega_{i}^{2} \tilde{\psi}_{i}+\dot{\alpha}(t) \omega_{i} \tilde{b}_{i}, & & i=1, \ldots, M, \\
\frac{\mathrm{d}^{2}}{\mathrm{~d} t^{2}} \tilde{b}_{i} & =-\alpha(t) \omega_{i}^{2} \tilde{b}_{i}, & i & =1, \ldots, M, \\
\frac{\mathrm{d}^{2}}{\mathrm{~d} t^{2}} \tilde{b}_{i} & =0, & i & =M+1, \ldots, N .
\end{aligned}
$$

To summarize, let $\boldsymbol{X}=\boldsymbol{Q} \boldsymbol{D}_{L}^{-1 / 2} \boldsymbol{S} \in \mathbb{R}^{M \times M}$ and $\boldsymbol{Y}=\boldsymbol{R} / N_{f} \in \mathbb{R}^{N \times N}$. The columns of $\boldsymbol{X}$ and $\boldsymbol{Y}$, denoted by $\left(\boldsymbol{X}_{1}, \ldots, \boldsymbol{X}_{M}\right)$ and $\left(\boldsymbol{Y}_{1}, \ldots, \boldsymbol{Y}_{N}\right)$, respectively, represent the normal modes of $\boldsymbol{\psi}$ and $\boldsymbol{b}$. Then the normal mode decomposition

$$
\boldsymbol{\psi}=\boldsymbol{X} \tilde{\psi}, \quad \boldsymbol{b}=\boldsymbol{Y} \tilde{\boldsymbol{b}},
$$

yields a system of $M$ independent systems (B 9)-(B 10), plus the $N-M$ trivial dynamics (B 11).

Remark. Note that if viscosity is included in the model, with viscosity parameter $v$, then (B 1) takes the form

$$
\left[\begin{array}{cc}
-\boldsymbol{L} & 0 \\
0 & \frac{1}{N_{f}^{2}} \boldsymbol{I}_{N}
\end{array}\right] \frac{\mathrm{d}}{\mathrm{d} t}\left(\begin{array}{l}
\boldsymbol{\psi} \\
\boldsymbol{b}
\end{array}\right)=\left[\begin{array}{cc}
\nu \boldsymbol{L}^{2} & \alpha(t) \boldsymbol{K} \\
-\boldsymbol{K}^{\mathrm{T}} & 0
\end{array}\right]\left(\begin{array}{l}
\boldsymbol{\psi} \\
\boldsymbol{b}
\end{array}\right) .
$$

By inverting the matrix on the left, this system is again a linear non-autonomous differential equation of the form

$$
\frac{\mathrm{d} f}{\mathrm{~d} t}=\boldsymbol{A}(t) \boldsymbol{f},
$$

for some time-dependent matrix $\boldsymbol{A}(t)$. Even if $\boldsymbol{A}(t)$ can be diagonalized, the similarity transformation that achieves this will typically be local in time, $\boldsymbol{A}(t)=$ $\boldsymbol{X}(t) \boldsymbol{D}_{\boldsymbol{A}}(t) \boldsymbol{X}(t)^{-1}$, and so one would not expect there to be a change of variables for which the dynamics decouples for all time. We can carry through the transformations used above in the inviscid case for (B 13), and (B 8) becomes

$$
\frac{\mathrm{d}}{\mathrm{d} t}\left(\begin{array}{c}
\tilde{\boldsymbol{\psi}} \\
\tilde{\boldsymbol{b}}
\end{array}\right)=\left[\begin{array}{cc}
\nu \boldsymbol{S}^{\mathrm{T}} \boldsymbol{D}_{\mathbf{L}} \boldsymbol{R} & \alpha(t) \boldsymbol{\Omega} \\
-\boldsymbol{\Omega}^{\mathrm{T}} & 0
\end{array}\right]\left(\begin{array}{c}
\tilde{\boldsymbol{\psi}} \\
\tilde{\boldsymbol{b}}
\end{array}\right),
$$

where we observe that the oscillators have become fully coupled through the (viscous) diagonal term in general.

\section{REFERENCES}

ARnold, V. I. 1989 Mathematical Methods of Classical Mechanics, 2nd edn. Springer.

BüHLER, O. \& Holmes-CERFON, M. 2011 Decay of an internal tide due to random topography in the ocean. J. Fluid Mech. 678, 271-293. 
Dintrans, B., Rieutord, M. \& VAldettaro, L. 1999 Gravito-inertial waves in a rotating stratified spherical shell. J. Fluid Mech. 398, 271-297.

Drijfhout, S. \& MAAS, L. R. M. 2007 Impact of channel geometry and rotation on the trapping of internal tides. J. Phys. Oceanogr. 37, 2740-2763.

Echeverri, P., Yokossi, T., Balmforth, N. J. \& PeAcock, T. 2011 Tidally generated internal-wave attractors between double ridges. J. Fluid Mech. 669, 354-374.

FRICKER, P. \& NEPF, H. 2000 Bathymetry, stratification, and internal seiche structure. J. Geophys. Res. 105, 14,237-14,251.

Gerkema, T. \& VAN HAREN, H. 2012 Absence of internal tidal beams due to non-uniform stratification. J. Sea Res. doi:10.1016/j.seares.2012.03.008.

Grisouard, N., Staquet, C. \& Pairaud, I. 2008 Numerical simulation of a two-dimensional internal wave attractor. J. Fluid Mech. 614, 1-14.

Hairer, E., Lubich, C. \& WAnNer, G. 2006 Geometric Numerical Integration: Structurepreserving Algorithms for Ordinary Differential Equations. Springer.

Hazewinkel, J., van Breevoort, P., Dalziel, S. B. \& MaAs, L. R. M. 2008 Observations on the wavenumber spectrum and evolution of an internal wave attractor. J. Fluid Mech. 598, 373-382.

Hazewinkel, J., Grisouard, N. G. \& Dalziel, S. B. 2011 Comparison of laboratory and numerically observed scalar fields of an internal wave attractor. Eur. J. Mech. B 30 (1), 51-56.

Hazewinkel, J., Tsimitri, C., MaAs, L. R. M. \& DAlziel, S. B. 2010 Observations on the robustness of internal wave attractors to perturbations. Phys. Fluids 22, 107102.

Holm, D. D., Marsden, J. E. \& Ratiu, T. S. 2002 The Euler-Poincaré equations in geophysical fluid dynamics. In Large-Scale Atmosphere-Ocean Dynamics II (ed. I. Roulstone \& J. Norbury), pp. 251-300. Cambridge University Press.

JoHn, F. 1941 The Dirichlet problem for a hyperbolic equation. Am. J. Math. 63, 141-154.

Kopecz, S. 2006 Fractal internal wave patterns in a tilted square. Unpublished report, Kassel University.

LAM, F.-P. A. \& MAAS, L. R. M. 2008 Internal wave focusing revisited: a reanalysis and new theoretical links. Fluid Dyn. Res. 40 (2), 95-122.

LAZERTE, B. D. 1980 The dominating higher order vertical modes of the internal seiche in a small lake. Limnol. Oceanogr. 25 (S), 846-854.

Leimkuhler, B. J. \& ReICH, S. 2004 Simulating Hamiltonian Dynamics. Cambridge University Press.

Lighthill, J. 1996 Internal waves and related initial-value problems. Dyn. Atmos. Oceans 23, $3-17$.

MAAs, L. R. M. 2005 Wave attractors: linear yet nonlinear. Intl J. Bifurcation Chaos 15 (9), 2757-2782.

MAAs, L. R. M. 2009 Exact analytic self-similar solution of a wave attractor field. Physica D: Nonlinear Phenomena 238 (5), 502-505.

MaAs, L. R. M., Benielli, D., Sommeria, J. \& LAM, F. P. A. 1997 Observation of an internal wave attractor in a confined, stably stratified fluid. Nature 388, 557-561.

MAAS, L. R. M. \& LAM, F.-P. A. 1995 Geometric focusing of internal waves. J. Fluid Mech. 300, $1-41$.

McEwan, A. D. \& Robinson, R. M. 1975 Parametric instability of internal gravity waves. J. Fluid Mech. 67 (4), 667-687.

MCLACHLAN, R. I. 1995 Symplectic integration of Hamiltonian wave equations. Numer. Math. 66 (1), 465-492.

Morrison, P. J. 1998 Hamiltonian description of the ideal fluid. Rev. Mod. Phys. 70 (2), $467-521$.

OGILVIE, G. I. 2005 Wave attractors and the asymptotic dissipation rate of tidal disturbances. J. Fluid Mech. 543, 19-44.

Olver, P. J. 1986 Applications of Lie Groups to Differential Equations. Springer.

Rieutord, M., Georgeot, B. \& VAldettaro, L. 2000 Wave attractors in rotating fluids: a paradigm for ill-posed Cauchy problems. Phys. Rev. Lett. 85, 4277-4280. 
Salmon, R. 1998 Lectures on Geophysical Fluid Dynamics. Oxford University Press.

Da Silva, J. C. B., Magalhães, J., Gerkema, T. \& MaAs, L. R. M. 2012 Internal solitary waves in the Red Sea: an unfolding mystery. Oceanography 25 (2), 96-107.

Swart, A., Sleijpen, G. L. G., MaAs, L. R. M. \& Brandts, J. 2007 Numerical solution of the two-dimensional Poincaré equation. J. Comput. Appl. Math. 200 (1), 317-341.

TANG, W. \& PEACOCK, T. 2010 Lagrangian coherent structures and internal wave attractors. Chaos 20, 017508.

Tilgner, A. 1999 Driven inertial oscillations in spherical shells. Phys. Rev. E 59, 1789-1794.

Whitham, G. B. 1999 Linear and Nonlinear Waves, 2nd edn. Wiley. 